\title{
Tumor Targeting Chemo- and Photodynamic Therapy Packaged in Albumin for Enhanced Anti-Tumor Efficacy
}

This article was published in the following Dove Press journal: International Journal of Nanomedicine

\author{
Ke Zheng $\mathbb{D}^{1-3}$ \\ Hongyan Liu' \\ Xinxin Liu' \\ Ying Wang' \\ Linlin $\mathrm{Li}^{2}$ \\ Shijie $\mathrm{Li}^{2}$ \\ Jinping $\mathrm{Xue}^{2}$ \\ Mingdong Huang (iD) ${ }^{2}$ \\ 'College of Chemical Engineering, \\ Qingdao University of Science and \\ Technology, Qingdao, Shandong 266042, \\ People's Republic of China; ${ }^{2}$ College of \\ Chemistry, Fuzhou University, Fuzhou, \\ Fujian 350I I8, People's Republic of \\ China; ${ }^{3}$ Key Laboratory of \\ Pharmaceutical Research for Metabolic \\ Disease, Qingdao University of Science \\ and Technology, Qingdao, Shandong \\ 266042, People's Republic of China
}

Purpose: Combination therapy for tumors is an important and promising strategy to improve therapeutic efficiency. This study aims at combining tumor targeting, chemo-, and photodynamic therapies to improve the anti-tumor performance.

Patients and Methods: Human serum albumin (HSA), as a nontoxic and biodegradable drug carrier, was used to load hydrophobic photosensitizers (mono-substituted $\beta$-4-pyridyloxy phthalocyanine zinc, mPPZ) by a dilution-incubation-purification (DIP) strategy to form molecular complex HSA:mPPZ. This complex was cross-linked as nanoparticles, and then chemotherapy drug doxorubicin (DOX) was adsorbed into the nanoparticles to achieve combined photodynamic therapy and chemotherapy. Next, the surface of the obtained composite was modified by a tumor surface receptor (urokinase receptor) targeting agent (ATF-HSA) using a noncovalent method to obtain the final product (ATF-HSA@HSA: mPPZ:DOX nanoparticles, AHmDN).

Results: AHmDN exhibited strong stability, remarkable cytotoxicity and higher uptake to tumor cells. Cell imaging analysis indicated that DOX was separated from AHmDN and uniformly distributed in cell nucleus while mPPZ localized in cytoplasm. The PDT activity of all the samples had been confirmed by the detection of intracellular ROS. In animal experiments, $\mathrm{AHmDN}$ was demonstrated to have a prominent tumor-targeting effect using a 3D imaging system. In addition, the enhanced antitumor effect of $\mathrm{AHmDN}$ in tumorbearing mice was also been observed. Importantly, the tumor-targeting effect of such nanoparticles lasted for about 14 days after one injection.

Conclusion: These albumin nanoparticles with combined functions of tumor targeting, chemotherapy and photodynamic therapy can highly enhance the anti-tumor effect. This drug delivery system can be applied to package other hydrophobic photosensitizers and chemotherapy drugs for improving therapeutic efficacy to tumors.

Keywords: drug delivery system, combination therapy, phthalocyanine, doxorubicin, urokinase receptor mediated targeting

\section{Introduction}

Cancer remains as a leading cause of death in humans and a major barrier limiting life expectancy in the 21 st century. ${ }^{1}$ Chemotherapy is a widely used treatment modality for cancer around the globe. Unfortunately, they also inhibit the rapid growth of bone marrow and gastrointestinal tract cells, which leads to serious side effects. ${ }^{2}$ In addition, varied therapeutic responses among different patients and the tendency to induce drug resistance limit the application of chemotherapeutic drugs. ${ }^{3}$
Correspondence: Ke Zheng

College of Chemical Engineering, Qingdao

University of Science and Technology, 53

Zhengzhou Road, Qingdao, Shandong

266042, People's Republic of China

Tel +86 132 10185127

Email zhengke_qust@163.com 
The improvement of the anti-cancer therapeutic effect has attracted persistent attention in basic research.

An important strategy to improve therapeutic efficiency is to combine multiple anti-cancer agent together or with other treatment modality. ${ }^{4-7}$ Simply mixing different therapeutic agents may not be suitable due to the difference in pharmacokinetic profiles and inconsistent tumor uptake. ${ }^{8}$ Many functional nanoparticles are developed to deliver various anticancer agents to tumor sites for a synergistic therapeutic effect. Such nanoparticles include gold nanoparticles, ${ }^{9}$ polymeric micelles, ${ }^{10,11}$ silica nanoparticles ${ }^{12,13}$ and magnetic nanoparticles. ${ }^{14,15}$ Nanoparticles can accommodate multiple drugs for combined chemotherapy, gene therapy, photothermal and photodynamic therapies to achieve a synergistic antitumor therapeutic effect. However, these materials are exogenous substances and may bring latent harm to the human body.

Human serum albumin (HSA) is an endogenous globular protein and is the most abundant protein in plasma ${ }^{16}$ with the characteristics of nontoxic, non-immunogenic and biodegradable. ${ }^{17}$ HSA is composed of three structurally similar domains (I, II, and III), each containing two subdomains (A and B). ${ }^{18}$ Some hydrophobic drugs generally bind to drug-binding pockets in subdomains IIA and IIIA, namely, Sudlow site I and site II. ${ }^{19}$ These sites have a strong propensity for hydrophobic drugs, which makes HSA molecules an ideal drug carrier. ${ }^{20}$

HSA nanoparticles can target tumor by EPR (enhanced permeability retention) effect. ${ }^{21}$ It is reported that receptormediated targeting is an important factor to further improve tumor targeting. ${ }^{22}$ Urokinase-type plasminogen activator receptor (UPAR) is overexpressed in many types of cancer, but not in most normal tissues. Amino-terminal fragment (ATF) is the primary receptor binding region of uPA which has a high affinity with $\mathrm{uPAR}^{23}$ ATF is often used to modify the surface of nanoparticles by covalent cross-linking. ${ }^{24}$ However, the covalent coupling method most likely interferes with the binding ability of ATF, which leads to the loss of targeting.

In our work, hydrophobic phthalocyanine zinc (monosubstituted $\beta$-4-pyridyloxy phthalocyanine zinc, abbreviated as $\mathrm{mPPZ}$ ) molecules, as photosensitizers for photodynamic therapy (PDT), were loaded into HSA using dilutionincubation-purification (DIP) method. This non-covalent package can inhibit the aggregation of $\mathrm{ZnPc}$ molecules and make them have a stronger fluorescence signal. HSA molecules loading disaggregated $\mathrm{mPPZ}$ were then formed as nanoparticles (HSA:mPPZ nanoparticles, abbreviated $\mathrm{HmN}$ ), which was further incorporated with a chemotherapeutic drug doxorubicin, leading to HSA:mPPZ:DOX nanoparticles (abbreviated HmDN). Next, the surface of HmDN was modified with tumor-targeting fusion protein ATF-HSA using strong hydrophobic drug paclitaxel (PTX) as a coupling agent for its affinity with HSA to form final product ATFHSA@HSA:mPPZ:DOX nanoparticles (abbreviated as AHmDN, HSA:mPPZ:DOX nanoparticles modified with HSA was abbreviated as $\mathrm{HHmDN}$ ). The schematic synthesis process was shown in Scheme 1. These nanoparticles were characterized by ultraviolet-visible, fluorescence spectrum, dynamic light scattering, scanning electron microscope, and were proved to have strong stability. AHmDN was found to have the highest cytotoxicity among all. Flow cytometry analysis demonstrated the tumor cell targeting property of $\mathrm{AHmDN}$ and more cells in late apoptotic or necrosis induced by $\mathrm{AHmDN}$. Cell imaging showed $\mathrm{mPPZ}$ distributed in

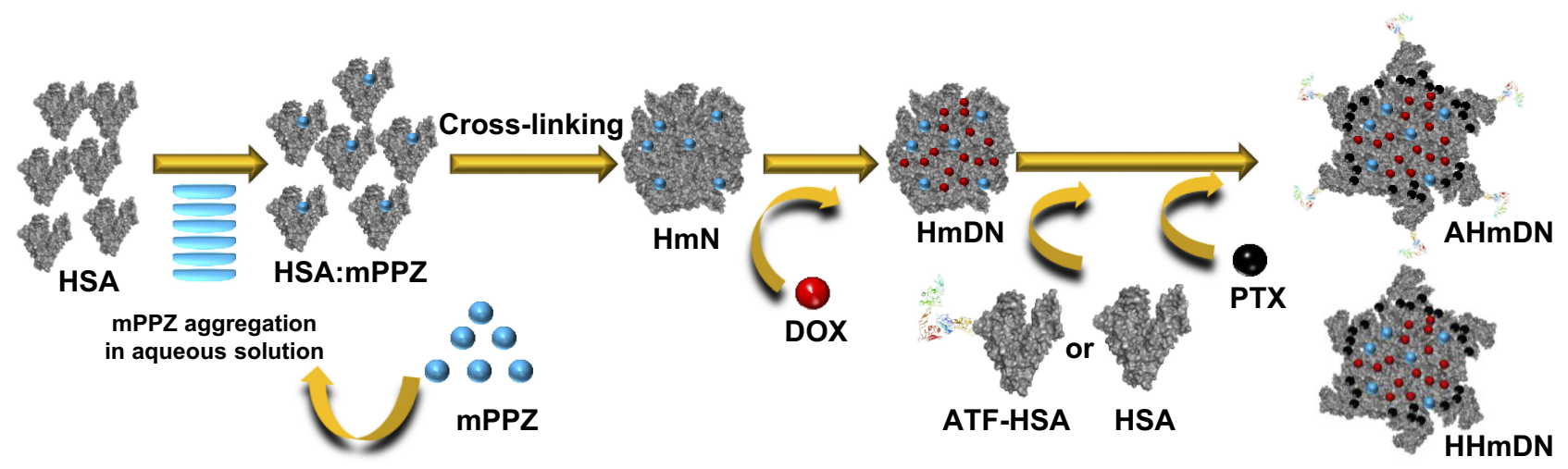

Scheme I Schematic illustration of the preparation of nanoparticles, HmN, HmDN, HHmDN and AHmDN.

Abbreviations: PDT, photodynamic therapy; ZnPc, zinc phthalocyanine; DIP, dilution-incubation-purification; mPPZ, mono-substituted $\beta$-4-pyridyloxy phthalocyanine zinc; HSA, Human serum albumin; DOX, doxorubicin; PTX, paclitaxel; HmN, HSA:mPPZ nanoparticles; HmDN, HSA:mPPZ:DOX nanoparticles; HHmDN, HSA@HSA:mPPZ: DOX nanoparticles; AHmDN, ATF-HSA@HSA:mPPZ:DOX nanoparticles. 
cytoplasm and DOX in cell nucleus after the incubation with these nanoparticles. Albumin packaging by DIP method made $\mathrm{mPPZ}$ have higher ROS production efficiency. AHmDN showed prominent tumor targeting and antitumor effect through in vivo experiments. Remarkably, these nanoparticles with non-aggregated $\mathrm{mPPZ}$ had a long targeting effect up to 14 days after an initial injection. This loading method and drug delivery system may be used for other hydrophobic photosensitizers and chemotherapy drugs for improved tumor targeting and antitumor effect.

\section{Materials and Methods Materials}

Photosensitizer mPPZ was synthesized and purified as per a previous method (synthesis procedure was shown in Scheme S1). ${ }^{25}$ DOX and PTX were purchased from Wuhan DKY Technology Co. Ltd. (Wuhan, People's Republic of China). HSA was purchased from Shanghai RAAS blood products co. Ltd. Diethylaminoethyl (DEAE) anion exchange resin was purchased from GE Healthcare (Uppsala, Sweden). Other chemicals were purchased either from Sigma-Aldrich (St Louis, MO, USA) or from Sinopharm Chemical Reagent Co. Ltd. (Shanghai, People's Republic of China). Non-smallcell lung carcinoma cells (H1299) and human embryo lung fibroblasts cells (HELF) were purchased from American Type Culture Collection (ATCC, Manassas, VA, USA). Mouse H22 hepatoma cells were from Cell Bank of Chinese Academy of Sciences (Shanghai, People's Republic of China). Clean level Kunming mice were purchased from Shanghai SLAC Laboratory Animal Co. Ltd., Chinese Academy of Sciences.

\section{Loading Disaggregated mPPZ Molecules into HSA}

HSA solution ( $1 \mathrm{~mL}$, containing 200mg HSA) was added into a mixed solution of $898 \mathrm{~mL} 20 \mathrm{mM}$ Tris- $\mathrm{HCl}$ ( $\mathrm{pH} 8.5$, containing $50 \mathrm{mM} \mathrm{NaCl}$ ) and $80.9 \mathrm{~mL}$ DMSO. Then, $10 \mathrm{~mL}$ DMSO solution of $\mathrm{mPPZ}(6.1 \mathrm{mg}$, with HSA and $\mathrm{mPPZ}$ at a molar ratio of 1:3) was dropwise added into the mixed solution (the final concentration of $\mathrm{mPPZ}$ was $10 \mu \mathrm{M}$ ) with stirring during the whole process. After stirring for $12 \mathrm{hrs}$ in the dark room, the resulting mixing solution was then applied to a DEAE anion exchange column pre-equilibrated with $20 \mathrm{mM}$ Tris- $\mathrm{HCl}$, $50 \mathrm{mM} \mathrm{NaCl}, \mathrm{pH} 8.5$. The column was then washed with 20mM Tris- $\mathrm{HCl}, 50 \mathrm{mM} \mathrm{NaCl}, \mathrm{pH}$ 8.5. HSA loading mPPZ was eluted with $20 \mathrm{mM}$ Tris- $\mathrm{HCl}, 300 \mathrm{mM} \mathrm{NaCl}, \mathrm{pH} 8.5$ and the complex HSA:mPPZ was collected. Finally, the solution of HSA:mPPZ was dialysed to PBS. The stoichiometric ratio of
HSA and mPPZ in the complex HSA:mPPZ was obtained by measuring the ratio of the concentrations of HSA and $\mathrm{mPPZ}$. HSA:mPPZ was disintegrated by $2 \%$ SDS. Then, the concentration of HSA was determined by a BCA Protein Assay Kit (BioTek Corporation, Beijing, People's Republic of China). The lysate was diluted into $90 \%$ DMSO solution and the concentration of $\mathrm{mPPZ}$ was determined using its fluorescent quantitation standard curve with its characteristic maximal emission wavelength $686 \mathrm{~nm}$ (excitation wavelength at $610 \mathrm{~nm})$.

\section{Preparation of HSA:MPPZ Nanoparticles Adsorbing DOX Molecules}

Ethanol was added into HSA:mPPZ solution $(3 \mathrm{~mL})$ with the concentration of HSA $10 \mathrm{mg} / \mathrm{mL}$ and the final concentration of ethanol was 55\%. This mixed solution was stirred for 10 mins and then $20 \mu \mathrm{L} 8 \%$ glutaraldehyde was added into them followed by stirring for $12 \mathrm{hrs}$ to get HSA nanoparticles loading $\mathrm{mPPZ}, \mathrm{HmN}$. After the dialysis to PBS, HmN was collected by centrifugation at $13000 \mathrm{rpm}$ for $10 \mathrm{mins}$. The precipitate was resuspended using $500 \mu \mathrm{L}$ PBS and then $25 \mu \mathrm{L}$ DMSO solution of DOX $(150 \mathrm{mM})$ was added into the resuspended solution. After mixing for $2 \mathrm{~h}$, DOX was attached to $\mathrm{HmN}$ and final product $\mathrm{HmDN}$ was collected and purified by centrifugation at $13000 \mathrm{rpm}$ for $10 \mathrm{mins}$.

\section{The Surface Modifying of $\mathrm{HmDN}$ by ATF-HSA}

The fusion protein ATF-HSA was constructed, expressed and purified as previously described. ${ }^{26}$ ATF-HSA (12.7mg, $200 \mu \mathrm{L})$ was added into the PBS solution of $\mathrm{HmDN}(1 \mathrm{~mL}$, containing 10mg HSA). After stirring for 30mins, DMSO solution of PTX $(10 \mathrm{mM}, 20 \mu \mathrm{L})$ was added into the mixed solution and then there was another stirring for $12 \mathrm{hrs}$. In the end, the final solution was centrifuged at 13000rpm for 10 mins to purify and collect the product AHmDN. HHmDN was prepared as the same method except substituting ATFHSA with HSA.

\section{Characterization of the Prepared Nanoparticles}

The ultraviolet-visible spectrum and fluorescence emission spectrum of mPPZ, HSA:mPPZ, HmN, HmDN, AHmDN and $\mathrm{HHmDN}$ in PBS was detected by a microplate reader (BioTek Instruments, Inc., Winooski, VT, USA). The particle size, polydispersity (PDI) and potential was continuously monitored for 3 weeks by Malvern Nanosizer ZS (Malvern 
Instrument, UK). The morphology of these nanoparticles was characterized by a field emission scanning electron microscopy (FESEM) (JSM-6700F, JEOL, Tokyo, Japan). Fluorescence quantum yields $\left(\Phi_{\mathrm{F}}\right)$ of the prepared nanoparticles and free $\mathrm{mPPZ}$ were calculated by the formula $\Phi_{\mathrm{F}}$ $=\Phi_{\mathrm{F}(\mathrm{std})} \times\left(\mathrm{F} \times \mathrm{A}_{\text {std }} \times \mathrm{n}^{2}\right) /\left(\mathrm{F}_{\text {std }} \times \mathrm{A} \times \mathrm{n}^{2}{ }_{\text {std }}\right)$, where $\mathrm{F}$ and $\mathrm{F}_{\text {std }}$ were the areas under the fluorescence curves (excited at $610 \mathrm{~nm}$ ) of the sample and the standard, respectively. A and $\mathrm{A}_{\text {std }}$ stood for the fluorescence intensity of the sample and the standard at the excitation wavelength, and $\mathrm{n}^{2}$ and $\mathrm{n}^{2}$ std were the refractive indices of solvents used for the sample and the standard, respectively. Unsubstituted ZnPc in DMF was used as the standard, and the $\Phi_{\mathrm{F}}$ of $\mathrm{ZnPc}$ was 0.28 .

Encapsulation efficiency (EE) of the prepared nanoparticles was determined by the formula $\mathrm{EE} \%=\mathrm{W}_{\mathrm{e}} / \mathrm{W}_{0} \times 100 \%$. $\mathrm{W}_{\mathrm{e}}$ represented the content of $\mathrm{DOX}$ or $\mathrm{mPPZ}$ loaded in nanoparticles and $\mathrm{W}_{0}$ represented the total content of DOX or $\mathrm{MPPZ}$ containing free and encapsulated components. The encapsulated drugs in nanoparticles were separated from free drugs by the centrifugation at $13000 \mathrm{rpm}$ for $10 \mathrm{mins}$. Loading efficiency (LE) was calculated by the formula $L E \%=M_{e} / M_{m}$ $\times 100 \% . \mathrm{M}_{\mathrm{e}}$ represented the weight of DOX or $\mathrm{mPPZ}$ in the nanoparticles and $\mathrm{W}_{\mathrm{m}}$ represented the total weight of prepared nanoparticles. The concentration of HSA and $\mathrm{mPPZ}$ was detected by above methods while the concentration of DOX was determined using its quantitation standard curve with maximum absorption wavelength at 490nm. The drug release property of $\mathrm{HmN}, \mathrm{HmDN}, \mathrm{AHmDN}$ and $\mathrm{HHmDN}$ was monitored. All the nanoparticles were stored in PBS solution at $4^{\circ} \mathrm{C}$. After 2 days, 4 days, 8 days, 14 days, 21 days, these solutions were centrifuged at $13000 \mathrm{rpm}$ for $10 \mathrm{mins}$ and the precipitate was resuspended. Then, the concentration of HSA, mPPZ and DOX in resuspension solution was detected as described above. The release percent (RE\%) was calculated by the formula $\mathrm{RE} \%=1-\mathrm{W}_{\mathrm{r}} / \mathrm{W}_{\mathrm{t}} \times 100 \% . \mathrm{W}_{\mathrm{r}}$ represented the content of $\mathrm{DOX}$ or $\mathrm{MPPZ}$ in precipitate after the storage and $\mathrm{W}_{\mathrm{t}}$ represented their initial content in nanoparticles before the storage.

\section{In vitro Cytotoxicity}

H1299 or HELF cells were cultured in Roswell Park Memorial Institute (RPMI) 1640 medium supplemented with $10 \%$ fetal calf serum at $37^{\circ} \mathrm{C}$ in a humidified incubator with $5 \% \mathrm{CO}_{2}$ atmosphere. The viability of cells was determined by trypan blue dye exclusion. Cells were maintained in logarithmic phase. H1299 or HELF cells were seeded into 96-well Costar ${ }^{\circledR}$ plates with 8000 cells in each well. After the attachment, cells were incubated with $\mathrm{HmN}, \mathrm{HmDN}$,
$\mathrm{AHmDN}, \mathrm{HHmDN}$ and $\mathrm{mPPZ}$ at various concentrations of $\mathrm{mPPZ}(0.01 \mu \mathrm{M}, 0.02 \mu \mathrm{M}, 0.05 \mu \mathrm{M}, 0.1 \mu \mathrm{M}, 0.2 \mu \mathrm{M}, 0.5 \mu \mathrm{M})$. After $24 \mathrm{hrs}$, medium was replaced by fresh medium without samples followed by an illumination for $1 \mathrm{~min}$ with an LED light source $(680 \mathrm{~nm}, 100 \mathrm{~mW}$, Sundynamic Inc., Qingdao, China) at a light fluence of $1.5 \mathrm{~J} / \mathrm{cm}^{2}$. After $24 \mathrm{hrs}$, viable cells were checked by MTT method. Each experiment was repeated three times with four replicates at each time point. Dark toxicity of cells was detected by the same way just without illumination. The cytotoxicity of free DOX and free $\mathrm{mPPZ}$ at the same concentrations with DOX and mPPZ in nanoparticles was detected by the same method as above.

\section{Flow Cytometry Analysis}

H1299 cells were seeded in the 24-well plates at a density of $5 \times 10^{4}$ per well and they were, respectively, incubated with $\mathrm{HmN}, \mathrm{HmDN}, \mathrm{AHmDN}$ and $\mathrm{HHmDN}$ for $12 \mathrm{hrs}$ at the equivalent concentration of $0.5 \mu \mathrm{M} \mathrm{mPPZ}$ before they were harvested by centrifugation at $100 \mathrm{~g}$ for $5 \mathrm{mins}$ and washed by PBS for three times. Then, the cells were resuspended by PBS and the cellular uptake was analysed by the fluorescence measurement of $\mathrm{MPPZ}$ through flow cytometry (Accuri C6; BD, Franklin Lakes, NJ, USA). The cellular uptake analysis of HELF cells was done with the same steps.

Cell apoptosis was measured using Annexin V-FITC (fluorescein isothiocyanate)/PI (propidium iodide) Apoptosis Assay Kit (MultiSciences Biotech Co. Ltd., Hangzhou, People's Republic of China). H1299 cells $\left(5 \times 10^{4}\right)$ were, respectively, incubated with $\mathrm{HmN}, \mathrm{HmDN}, \mathrm{AHmDN}$ and $\mathrm{HHmDN}$ (at a final concentration of $\mathrm{mPPZ} 0.5 \mu \mathrm{M}$ ) for $12 \mathrm{hrs}$. Then, medium was replaced by fresh medium and cells were illuminated at a light fluence of $1.5 \mathrm{~J} / \mathrm{cm}^{2}$. After another $12 \mathrm{hrs}$, H1299 cells were harvested by centrifugation at $100 \mathrm{~g}$ for 5 mins and washed by Hanks balanced saline solution for three times. The cells were then resuspended in $200 \mu \mathrm{L}$ of staining buffer and stained by Annexin V-FITC $(2 \mu \mathrm{L})$ and PI $(4 \mu \mathrm{L})$ for 5 mins in the dark, followed by an analysis on the flow cytometer. The percentage of early apoptotic, and late apoptotic or necrotic cells was determined. Each experiment was repeated three times with four replicates at each time point.

\section{Cellular Localization}

The suspended cells (H1299) were seeded into confocal chamber slides (Nest Biotechnology Co. Ltd., Wuxi, China) and incubated at $37^{\circ} \mathrm{C}$ for $24 \mathrm{hrs}$. Then, $\mathrm{HmN}$, $\mathrm{HmDN}$, AHmDN or HHmDN were added into the 
medium with final concentration of $\mathrm{mPPZ} 0.5 \mu \mathrm{M}$. After incubation for $12 \mathrm{hrs}$, adherent cells were washed by PBS to remove unbound nanoparticles and then incubated in medium containing DNA fluorescent dye, DAPI (4'6-diamidino-2-phenylindole, $5 \mu \mathrm{g} / \mathrm{mL}$ ) for 30 mins. After another wash by PBS, fresh medium was added into the confocal chamber slides and the cells were imaged by Olympus FluoViewTM FV1000 laser scanning confocal microscope (Olympus Corp., Tokyo, Japan). The fluorescence of DOX or mPPZ in the cells was excited by an argon-ion laser light ( $\lambda=488 \mathrm{~nm}$ or $633 \mathrm{~nm}$, respectively), while the emitted fluorescence was filtered with barrier filters $(590 / 30 \mathrm{~nm}$ or $640-700 \mathrm{~nm}$ band pass, respectively). The fluorescence of DAPI was excited by diode laser $(\lambda=405 \mathrm{~nm})$ and the emitted fluorescence filtered with barrier filters $450 / 30 \mathrm{~nm}$ band pass. All parameters, including the laser line intensity, photometric gain, settings of photo-multiplier tube and filter attenuation, were kept constant throughout the entire imaging experiment. All images were analysed by Olympus Fluoview v2.1 software.

\section{Assessment of Intracellular ROS Levels}

After the H1299 cells adhered in confocal chamber slides, $\mathrm{HmN}, \mathrm{HmDN}, \mathrm{AHmDN}$ or $\mathrm{HHmDN}$ was added into the medium with the final concentration of $\mathrm{mPPZ} 0.5 \mu \mathrm{M}$. After the incubation for $12 \mathrm{hrs}$, cells were washed by PBS and then incubated with $10 \mu \mathrm{mol} / 12^{\prime}, 7^{\prime}$-dichlorofluorescein diacetate (DCFH-DA) solution (S0033, Beyotime, China) for $30 \mathrm{mins}$ in serum-free medium in the dark. The illumination $(680 \mathrm{~nm})$ at a light fluence of $1.5 \mathrm{~J} / \mathrm{cm}^{2}$ was followed by ROS Assessment. The fluorescence, based on the oxidative conversion of DCFH-DA to dichlorofluorescein (DCF) by ROS, was detected using Olympus FluoViewTM FV1000 laser scanning confocal microscope (Olympus Corp., Tokyo, Japan) with the excitation at $488 \mathrm{~nm}$. All images were analysed by Olympus Fluoview v2.1 software.

\section{Establishment of Hepatoma-22 $(\mathrm{H} 22)$ Tumor-Bearing Mice Model}

Female Kunming mice (4 weeks old and weighing 18$22 \mathrm{~g}$ ) were fed in accordance with the recommendations of the institutional animal care and use committee. Mouse $\mathrm{H} 22$ cells $\left(5.0 \times 10^{6}\right)$ were injected into mice abdominal cavity. After 6 days, mice were executed by breaking the neck and ascetic fluid was collected. Then, the $\mathrm{H} 22$ cells $\left(2.0 \times 10^{6}\right)$ from the ascetic fluid in normal saline $(200 \mu \mathrm{L})$ were subcutaneously injected on the back of each mouse. Typically, the mice were used for animal experiment in 5-7 days as the volume of tumor reached around $50 \mathrm{~mm}^{3}$. All animal experiments were approved by Institutional Animal Care Committee of Qingdao University of Science and Technology, and all animal studies were performed in compliance with the guidelines of the committee.

\section{Fluorescence Imaging in vivo}

The H22 tumor-bearing Kunming mice were randomly divided into four groups (five mice per group) with the equivalent average starting tumor size $\left(50 \mathrm{~mm}^{3}\right)$ and body weight $(23 \mathrm{~g})$. Normal saline solution of $\mathrm{HmN}, \mathrm{HmDN}$,

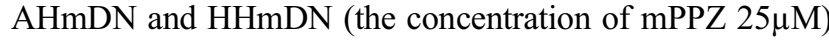
was, respectively, injected into the mice of four groups from caudal vein as the dose of $\mathrm{mPPZ}$ at $0.2 \mu \mathrm{mol} / \mathrm{kg}$. Then, these mice were fed in dark room and were imaged at $4 \mathrm{hrs}, 8 \mathrm{hrs}$, 16 hrs, 1 day, 1.5 days, 2 days, 3 days, 4 days, 5 days, 6 days, 8 days, 10 days, 12 days and 14 days with $\mathrm{mPPZ}$ as a probe. Isoflurane gas anesthesia system and fluorescent molecular tomography (FMT) $2500^{\mathrm{TM}}$ LX instrument (PerkinElmer, Waltham, MA, USA) were used for fluorescence imaging. For the determination of $\mathrm{mPPZ}$ concentration in mice, $0.2 \mu \mathrm{M}$ $\mathrm{mPPZ}$ solution in PBS containing 5\% Cremolphore EL was used as a standard to calibrate the instrument. The collected data were reconstructed by the software TrueQuant v3.0 (PerkinElmer, Waltham, MA, USA) to three dimensions, and the quantitative information was analysed by creating ROIs around the tumor site after the subtraction of fluorescence background.

\section{Antitumor Effect in vivo}

The H22 tumor-bearing mice with equivalent tumor volume $\left(50 \mathrm{~mm}^{3}\right)$ and weight $(23 \mathrm{~g})$ were randomly divided into five groups (10 mice per group) as described above. The normal saline solution of $\mathrm{HmN}, \mathrm{HmDN}, \mathrm{AHmDN}$ and HHmDN was, respectively, injected into the mice of four groups at the dose of $\mathrm{mPPZ} 0.2 \mu \mathrm{mol} / \mathrm{kg}$ using blank normal saline in the fifth group as a control. Then, all the mice were fed in dark room and the tumor site was illuminated by a $680 \mathrm{~nm}$ light source $(1 \mathrm{~W}$, Luma Care Medical Group, newport Beach, CA, USA) for 3 mins with a light dose of $50 \mathrm{~J} / \mathrm{cm}^{2}$. The PDT was implemented every day and lasted for 7 days. The tumor volume of every mouse was measured every day by a caliper through an ellipsoid volume formula, $\Pi / 6 \times \mathrm{s}$ (length $\times$ width $\times$ height). Every day, the weight of mice was measured using an electronic scale. 
After 7 days, all the mice of every experimental group were executed. Their tumor was stripped out and the tumor weight was recorded. In addition, H22 tumor-bearing mice were randomly divided into another five groups (12 mice per group) and were treated with $\mathrm{HmN}, \mathrm{HmDN}, \mathrm{AHmDN}$ or HHmDN, respectively, as above same method. After the therapy for 7 days, the number of live mice was recorded until 40 days.

\section{Statistical Analysis}

All data represented group means and standard errors of the mean (SEM). The experimental data in vitro and in vivo were analysed using the unpaired, two-tailed Student $t$-test. Differences at the $95 \%$ confidence level $(p<0.05)$ were considered to be statistically significant.

\section{Results}

\section{Characterization of Prepared Nanoparticles}

The aggregation behavior of MPPZ was monitored by its electronic spectrum. Figure S1A showed the typical absorption spectrum of zinc phthalocyanine $\mathrm{mPPZ}$ which has the maximum absorption peak at $670 \mathrm{~nm}$, demonstrating a monomer state of mPPZ in DMF. However, the monomer absorption peak was relatively reduced and the absorption peak at $630 \mathrm{~nm}$ was raised when the solution medium was substituted by water (Figure 1A) showing that $\mathrm{mPPZ}$ was in a state of aggregation. Through the package of HSA molecules, the monomer absorption peak of $\mathrm{MPPZ}$ was significantly enhanced in the complex HSA:mPPZ (Figure 1A), which demonstrated the disaggregation function of HSA by DIP method. In addition, the fluorescence emission intensity of HSA:mPPZ increased by about 10 times than $\mathrm{mPPZ}$ at the same concentration in PBS (Figure 1B). Though the fluorescence quantum yield $\Phi_{\mathrm{F}}$ of $\mathrm{HmN}, \mathrm{HmDN}, \mathrm{HHmDN}$ and $\mathrm{AHmDN}$ was a little lower than $\mathrm{mPPZ}$ in DMF, that was much higher than single $\mathrm{mPPZ}$ in PBS (Table S1). Therefore, the package of $\mathrm{mPPZ}$ inside HSA greatly improved its imaging capability.

The morphology of $\mathrm{HmN}$ and $\mathrm{HmDN}$ was measured by FESEM, which showed a spherical structure with size in accordance with the results of DLS data (Figure 1C). Compared to $\mathrm{HmN}$ and $\mathrm{HmDN}$, the morphology of AHmDN and HHmDN was slightly different and showed a shell structure with a rough surface, suggesting the surface coating of HSA or ATF-HSA (Figure S2). The absorption spectra of $\mathrm{HmN}, \mathrm{HmDN}, \mathrm{AHmDN}$ and $\mathrm{HHmDN}$ were shown in Figure 1D. HmDN, HHmDN and AHmDN had the similar absorption peaks including both characteristic absorption peak of DOX (Figure S1A) and mPPZ, which illustrated DOX molecules were loaded into the nanoparticles. It was worth noting that $\mathrm{mPPZ}$ still had an obvious monomer peak in the four nanoparticles, which indicated mPPZ was maintained being packaged inside HSA during their preparation process. The particle size of HSA:mPPZ was about $7 \mathrm{~nm}$ approximating the particle size of a single HSA molecule (Figure 1E), which signified no aggregation between HSA molecules induced by mPPZ. The particle size of $\mathrm{HmN}$ was increased to $98 \mathrm{~nm}$ through covalent crosslinking by glutaraldehyde. The particle size of HmDN was $105 \mathrm{~nm}$ nearly identical to $\mathrm{HmN}$. The surface modification by ATF-HSA or HSA mediated by PTX made the particle size of $\mathrm{HHmDN}$ and $\mathrm{AHmDN}$ further increase to $166 \mathrm{~nm}$ and $179 \mathrm{~nm}$, respectively (Figure 1E, Table S2). The small PDI value demonstrated that all prepared nanoparticles had high homogeneity (Table S2). The zeta potential of $\mathrm{HmN}, \mathrm{HmDN}$, AHmDN and $\mathrm{HHmDN}$ was identified at $-22 \mathrm{mV},-25 \mathrm{mV}$, $-33 \mathrm{mV}$ and $-30 \mathrm{mV}$, respectively (Figure $1 \mathrm{~F}$, Table S2), indicating their high colloidal stability in PBS solution. The $\mathrm{EE} \%$ value of four nanoparticles was about $13 \%$ for $\mathrm{mPPZ}$ and $60 \%$ for DOX, and the LE\% value was relatively small for the large HSA weight (Table S2).

During the storage at $4{ }^{\circ} \mathrm{C}$ for 3 weeks, the particle sizes and PDI of $\mathrm{HmN}, \mathrm{HmDN}, \mathrm{AHmDN}$ and $\mathrm{HHmDN}$ in PBS remained almost unchanged (Figures S3A and S3B), especially $\mathrm{AHmDN}$ and $\mathrm{HHmDN}$, where their surface was modified by a new method, maintained high stability during the storage process. The drugs should not leak from nanoparticles in the storage and before reaching the target organs. There was little RE\% of mPPZ for the four nanoparticles (less than 2\%) after the storage for $72 \mathrm{hrs}$, and the average RE\% of DOX for both $\mathrm{HHmDN}$ and $\mathrm{AHmDN}$ was $1.6 \%$ which was less than that for HmDN (Table S2). If $\mathrm{mPPZ}$ or DOX was released from the nanoparticles, the stoichiometric ratio with HSA could make a difference. Thus, the stoichiometric ratio of mPPZ:HSA and DOX: HSA was used to monitor drugs release from the $\mathrm{HmN}$, $\mathrm{HmDN}, \mathrm{HHmDN}$ and $\mathrm{AHmDN}$. The stoichiometric ratio of mPPZ:HSA had hardly changed in all four nanoparticles during the storage for 3 weeks (Figure S3C). These results demonstrated that the package of HSA for $\mathrm{mPPZ}$ was very tight and the formation of these nanoparticles did not affect the package effect. Figure S3D showed that HmDN had a slight release of DOX during the monitoring for 21 days. Using HmDN as a control, surface modification was shown 

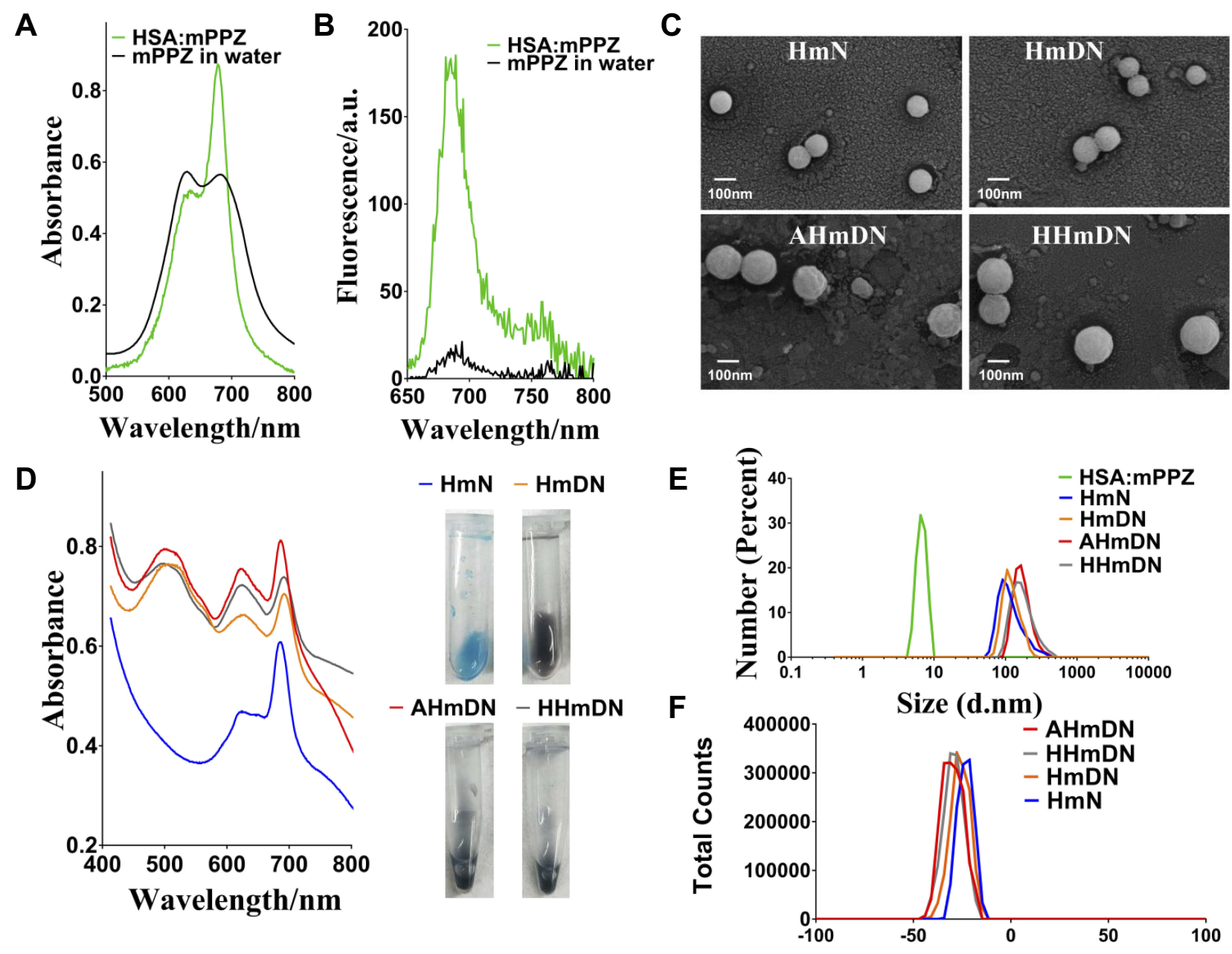

Apparent Zeta Potential (mV)

Figure I The characterization of the prepared nanoparticles.

Notes: (A) mPPZ in HSA:mPPZ prepared by DIP method was mainly in monomer form in PBS. (B) HSA:mPPZ had a stronger fluorescence peak than mPPZ in water solution. (C) Spherical structure of HmN, HmDN, HHmDN, AHmDN was shown in the FESEM images. (D) The ultraviolet-visible spectrums of HmN, HmDN, HHmDN, AHmDN. All mPPZ in nanoparticles was still in the monomer state after their preparation and the characteristic absorption peak at 490nm indicated DOX was loaded into $\mathrm{HmDN}, \mathrm{HHmDN}, \mathrm{AHmDN}$ nanoparticles. Illustrations were photographs of HmN, HmDN, HHmDN, AHmDN in PBS solution. (E) Hydrodynamic diameters of HSA: mPPZ, HmN, HmDN, HHmDN, AHmDN measured by DLS. (F) The zeta potential of HmN, HmDN, HHmDN, AHmDN.

to limit the release of DOX from HHmDN and AHmDN and render them excellent stability (Figure S3D).

\section{Cytotoxicity}

Since it is significant to clarify whether the package of HSA affected the activity of mPPZ and DOX. H1299 and HELF cells were used to evaluate the cytotoxicity. Figure 2 shows that $\mathrm{HmN}$ had barely cytotoxicity without illumination in both H1299 and HELF cells until the concentration of mPPZ went to $0.5 \mu \mathrm{M}$, which suggested the drug carrier was harmless for cells. HmN showed enhanced cytotoxicity with illumination, demonstrating the photodynamic activity of mPPZ. As DOX molecules were loaded into $\mathrm{HmN}$, both phototoxicity and dark toxicity of HmDN to the two cell lines were further increased, indicating combined antitumor effect of PDT and chemotherapy. Through the surface modification by HSA or ATF-HSA mediated by PTX, AHmDN and HHmDN still remained powerful cytotoxicity to both H1299 and HELF cells. PTX, as a chemotherapeutic drug, could stabilize microtubule and induced cytotoxicity by cutting off cell cycle in the G2/M phase, ${ }^{27}$ which may result in the increased toxicity for $\mathrm{HHmDN}$ and $\mathrm{AHmDN}$. It was worth noting that $\mathrm{AHmDN}$ had higher phototoxicity than HHmDN for H1299 cells with about triple difference at the mPPZ concentration of $0.1 \mu \mathrm{M}$, $0.2 \mu \mathrm{M}$ and $0.5 \mu \mathrm{M}$, while a similar phototoxicity for HELF cells (Figure 2). This may be attributed to a large amount of the 


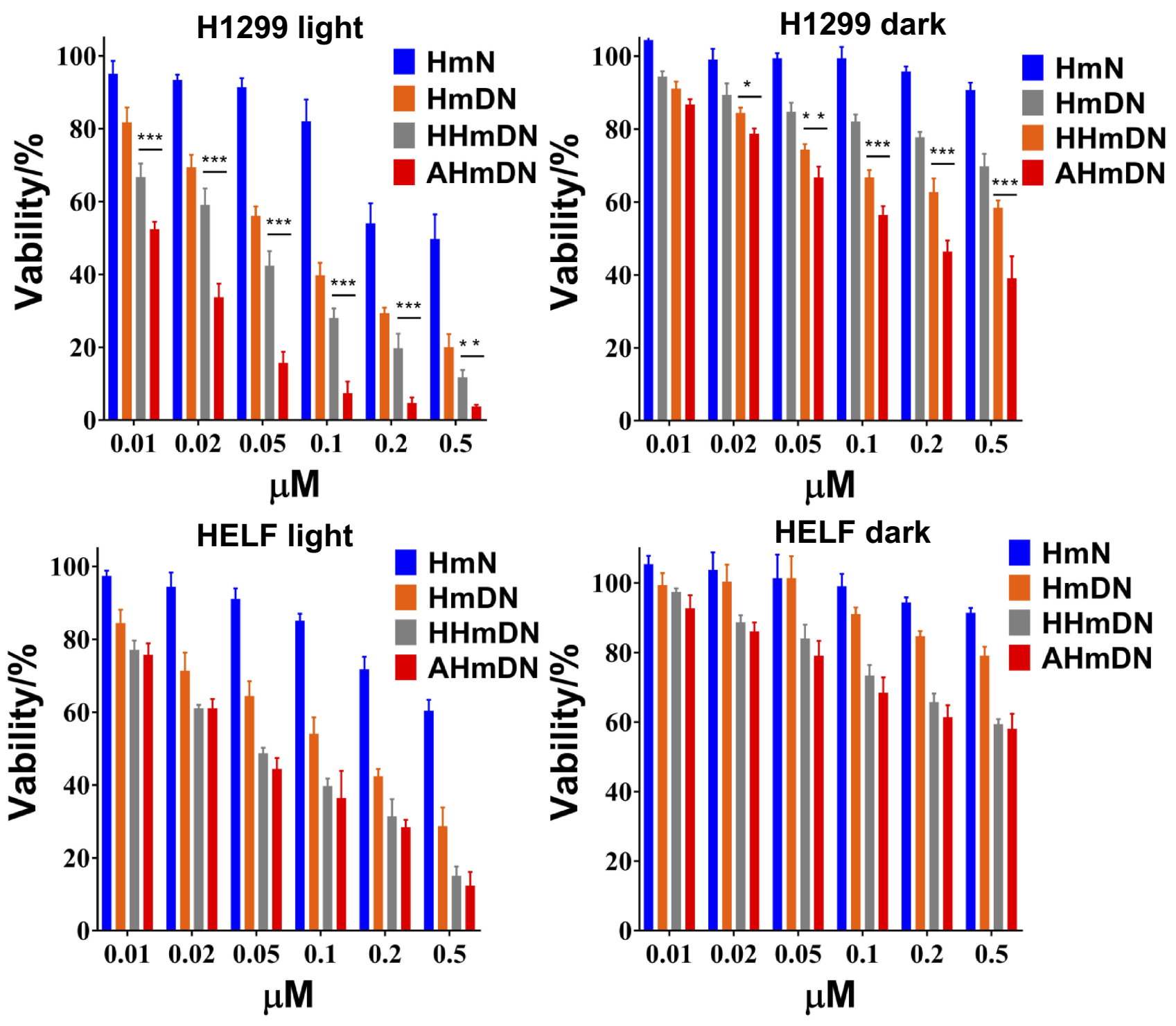

Figure 2 The cytotoxicity of $\mathrm{HmN}, \mathrm{HmDN}, \mathrm{HHmDN}, \mathrm{AHmDN}$ in HI299 and HELF cells.

Notes: HI299 and HELF cells were incubated with HmN, HmDN, HHmDN or AHmDN for 24hrs and cytotoxicity was detected by MTT method. Their dark toxicity illustrated the chemotherapy effect and the enhanced cytotoxicity with illumination $\left(1.5 \mathrm{~J} / \mathrm{cm}^{2}\right)$ showed the PDT effect. No matter dark toxicity or phototoxicity, AHmDN had almost similar cytotoxicity with HHmDN in HELF cells while AHmDN had about twofold higher cytotoxicity than HHmDN in HI299 cells, demonstrating the tumortargeting capability. $* * * \mathrm{P}<0.00 \mathrm{I}, * * \mathrm{P}<0.0 \mathrm{I}, * \mathrm{P}<0.05$.

uPAR receptor expressed on the H1299 cell surface whereas little UPAR expression on HELF cell surface. ${ }^{28}$ The difference in cytotoxicity demonstrated the targeting ability of AHmDN and there was no influence on the activity of ATF-HSA during the whole preparation process. Using the free $\mathrm{mPPZ}$ and free DOX at the same concentration with that in nanoparticles as the control, the results further demonstrated that combined treatment caused higher cytotoxicity than the treatment with single drug (Figure S4).

\section{Flow Cytometry}

In order to determine the targeting ability of ATF, the fluorescence intensity of $\mathrm{mPPZ}$ was evaluated to determine the amount of nanoparticles ingested by H1299 or HELF cells. After the incubation with $\mathrm{HmN}, \mathrm{HmDN}, \mathrm{AHmDN}$ or HHmDN for $12 \mathrm{hrs}$, H1299 or HELF cells were washed to remove unabsorbed nanoparticles and detected using flow cytometry. Figure $3 \mathrm{~A}$ shows that there was little variation in fluorescence intensity of $\mathrm{HmDN}, \mathrm{HHmDN}$ and $\mathrm{AHmDN}$ groups in HELF cells. However, the fluorescence intensity of AHmDN group in H1299 cells was higher than that of HmDN and HHmDN groups. The overexpression of uPAR receptor on $\mathrm{H} 1299$ cell surface than HELF cells may attribute to the higher uptake.

After the incubation with $\mathrm{HmN}, \mathrm{HmDN}, \mathrm{AHmDN}$ or HHmDN for $12 \mathrm{hrs}, \mathrm{H} 1299$ cells were illuminated by a LED 
A

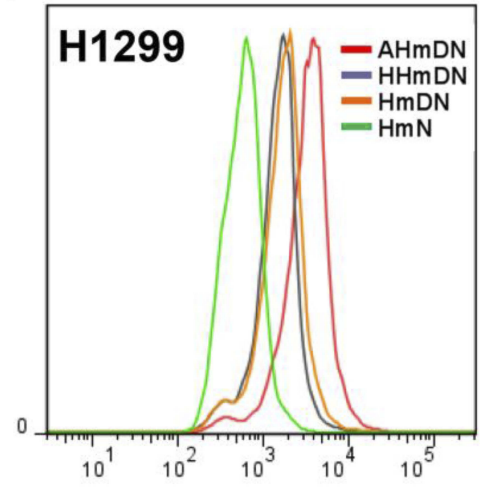

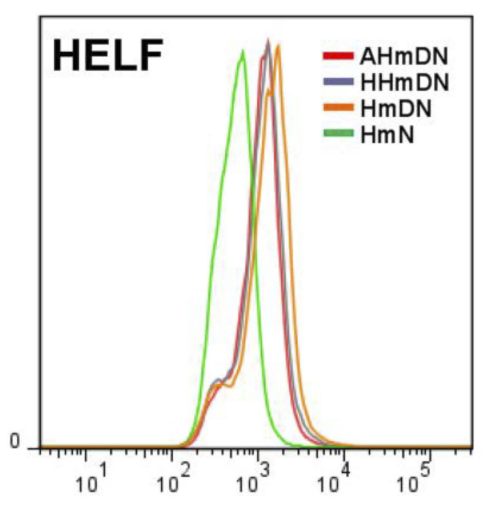

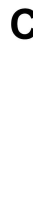

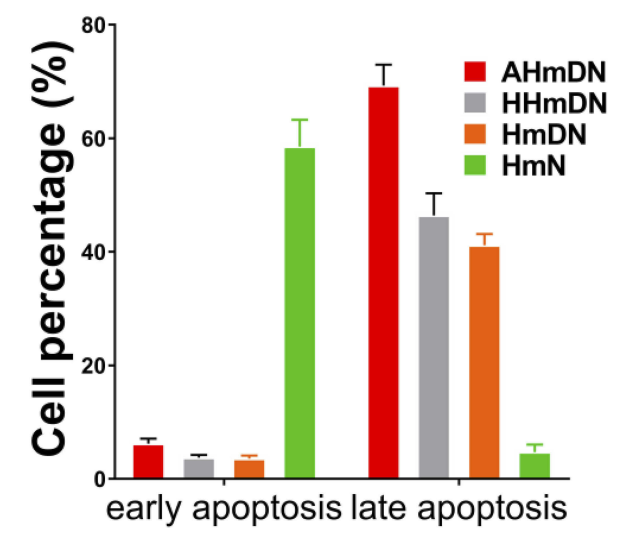
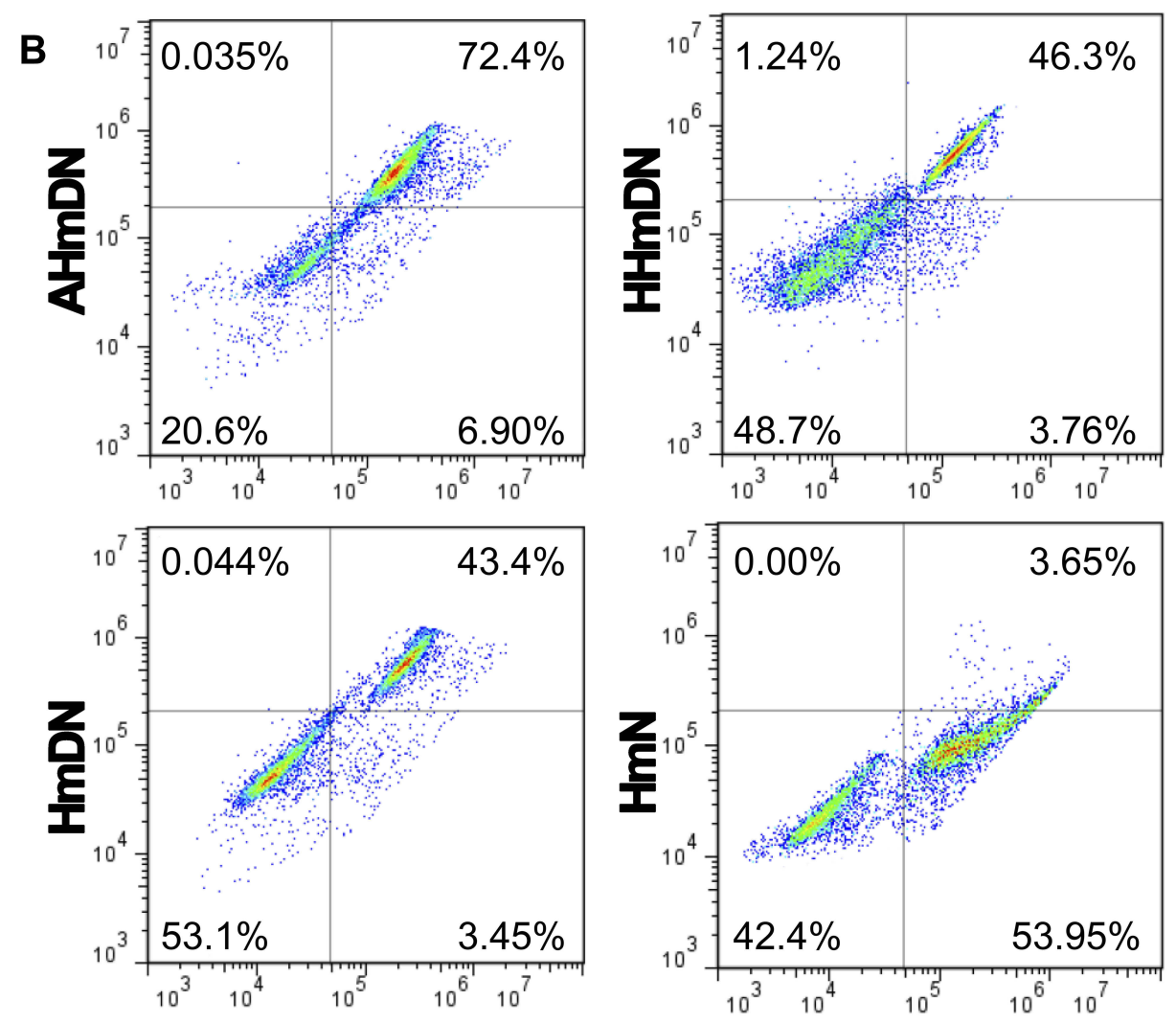

Figure 3 The cellular uptake and apoptosis analysis by flow cytometry.

Notes: (A) The cellular uptake analysis by flow cytometry in $\mathrm{HI} 299$ and HELF cells after the incubation with HmN, HmDN, HHmDN, AHmDN (the final concentration of mPPZ $0.5 \mu \mathrm{M}$ ) for $12 \mathrm{hrs}$. In HI299 cells, stronger fluorescence was detected in AHmDN group which showed enhanced cellular uptake of AHmDN demonstrating the tumor-targeting effect. (B) Flow cytometric distribution of $\mathrm{HI} 299$ cells stained by FITC-labeled Annexin $\mathrm{V}$ and PI. HI299 cells were illuminated at a light fluence of I.5 J/cm ${ }^{2}$ after the incubation with $\mathrm{HmN}, \mathrm{HmDN}, \mathrm{HHmDN}$ or AHmDN for $12 \mathrm{hrs}$. After I2hrs, the HI299 cells were collected and analyzed by flow cytometry. (C) Quantifications of the cell population showed that $\mathrm{HmDN}, \mathrm{HHmDN}$ and $\mathrm{AHmDN}$ groups had more necrotic or late apoptotic cells than early apoptotic cells while $\mathrm{HmN}$ group had more early apoptotic cells, which was likely due to a different cytotoxicity mechanism.

light source at $680 \mathrm{~nm}$. Followed by apoptosis for another $12 \mathrm{hrs}$, H1299 cells were stained with FITC-label Annexin $\mathrm{V}$ and PI for a flow cytometry analysis. High Annexin V and low PI staining were an indication of early apoptosis of cells, whereas strong staining of both Annexin V and PI indicated the cells were in the stage of late apoptosis or necrosis. Figure 3B shows that more H1299 cells in HmN group were in the state of early apoptosis, which also was demonstrated through the quantifications of the cell population (Figure 3C). However, AHmDN, HHmDN and HmDN groups had very little cells in early apoptosis accompanied by more cells in late apoptotic or necrosis. AHmDN, HHmDN and HmDN probably had different cell death mechanism compared with $\mathrm{HmN}$ due to the incorporation of chemotherapy drugs. Moreover, although there was almost the same early apoptosis cell proportion in $\mathrm{HHmDN}$ and $\mathrm{HmDN}$ groups, HHmDN group had 
a slightly higher distribution of cells in late apoptosis or necrosis than HmDN group. AHmDN group had more cells in early apoptosis and almost 1.5-fold more cells in late apoptosis or necrosis than $\mathrm{HHmDN}$ or $\mathrm{HmDN}$ group, which further validated the enhanced cytotoxicity of AHmDN.

\section{Subcellular Localization}

Could the DOX in HmDN, AHmDN and HHmDN enter into cell nucleus? The diffusion range of ROS generated by photosensitizers was limited to less than $0.02 \mu \mathrm{m}^{29}$ and the intracellular distribution of photosensitizers is key to their photodynamic activity. Thus, we imaged the H1299 cells to monitor the distribution of mPPZ and DOX in cells after the cells were incubated with $\mathrm{HmN}, \mathrm{HmDN}, \mathrm{AHmDN}$ or $\mathrm{HHmDN}$ for $12 \mathrm{hrs}$ at the concentration of $\mathrm{mPPZ} 0.5 \mu \mathrm{M}$. The fluorescence of $\mathrm{mPPZ}$ (red) showed that $\mathrm{HmDN}$, AHmDN and HHmDN were ingested into H1299 cells and mPPZ mainly distributed in the cytoplasm with almost no overlap at cell nucleus (blue fluorescence) (Figure 4). mPPZ in HmN group distributed in cytoplasm similarly (Figure S5). It was noteworthy that DOX molecules almost completely localized to the nucleus based on the analysis of intracellular distribution of fluorescence intensity (Figure 4). This DOX localization at nucleus is consistent with its widely accepted mechanism of intercalating with DNA, disrupting the replication and transcription processes, finally leading to cell death. $^{30}$ The differential distribution of $\mathrm{mPPZ}$ and DOX suggested DOX may be released from nanoparticles.

\section{Intracellular ROS Detection}

The PDT effect for H1299 cells killing by AHmDN, HHmDN, HmDN and $\mathrm{HmN}$ was attributed to ROS. Therefore, intracellular ROS was evaluated through the DCF fluorescence converted by the oxidation of DCFHDA. After absorbing AHmDN, HHmDN, HmDN or $\mathrm{HmN}$, cells were incubated with DCFH-DA solution and then illuminated at a light fluence of $1.5 \mathrm{~J} / \mathrm{cm}^{2}$. Cell images were collected by laser scanning confocal microscope. In Figure S6, cell nucleus localization was determined by the fluorescence of Hoechst 33342 (blue), and ROS generated from $\mathrm{AHmDN}, \mathrm{HHmDN}, \mathrm{HmDN}, \mathrm{HmN}$ or mPPZ was determined by the fluorescence of DCF (green). All the H1299 cells absorbing AHmDN, HHmDN, HmDN or HmN showed obvious DCF fluorescence, while the cells treated with mPPZ had very weak fluorescence of DCF. These results demonstrated that HSA nanoparticles loading mPPZ by DIP method had higher ROS production efficiency than mPPZ molecules in the cells. This was because that $\mathrm{mPPZ}$ in HSA was disaggregated by DIP method and mPPZ in monomer form had a more efficient ROS production rate than that in aggregation form.

\section{Tumor Targeting}

An ideal drug delivery system should transport drugs to target organs. After injecting $\mathrm{HmN}, \mathrm{HmDN}, \mathrm{AHmDN}$ or $\mathrm{HHmDN}$ into $\mathrm{H} 22$ tumor-bearing mice as the dose of $\mathrm{mPPZ}$ at $0.2 \mu \mathrm{mol} / \mathrm{kg}$, the tumor targeting was determined by a 3D animal imaging system. All the nanoparticles were detected to accumulate gradually at the tumor site using normal tissues as control (Figure 5A). Quantitation results (Figure 5B) indicated that there was almost the same amount of $\mathrm{mPPZ}$ in $\mathrm{HmDN}$ and $\mathrm{HmN}$ groups after the injection for 7 days. However, HmDN group had more $\mathrm{mPPZ}$ at tumor site than $\mathrm{HmN}$ group after 7 days, which was consistent with the result of cell uptake. It was worth noting that HHmDN group had higher content of $\mathrm{mPPZ}$ than both $\mathrm{HmDN}$ and $\mathrm{HmN}$ groups at any point of time and this distinction was continued for 14 days. What is more, the mice images indicated that $\mathrm{AHmDN}$ group had stronger fluorescence intensity at tumor site than $\mathrm{HHmDN}$ group throughout the detection process and the amount of $\mathrm{mPPZ}$ in $\mathrm{AHmDN}$ group was 1.5 times more than that in HHmDN group at the 14th day (Figure 5B). Supplementary video of mice after the treatment with AhmDN (Video 1) and HHmDN (Video 2) for 14 days further demonstrated the higher content of mPPZ of AHmDN group than that of HHmDN group from multiple angles. This further validated the tumor targeting of AHmDN by modifying ATF-HSA on the surface. The percentage of the $\mathrm{mPPZ}$ at the tumor site in the whole body was kept increasing which was consistent with the results from Figure 5B (Figure 5C). Particularly on the 14th day, the percentage of the $\mathrm{MPPZ}$ at the tumor site in AHmDN group was risen to approximately $30 \%$, and the high content was very beneficial for antitumor treatment.

\section{Antitumor Effect}

$\mathrm{HmN}, \mathrm{HmDN}, \mathrm{AHmDN}$ or $\mathrm{HHmDN}$ was, respectively, injected into $\mathrm{H} 22$ tumor-bearing mice with equivalent tumor volume $\left(50 \mathrm{~mm}^{3}\right)$ and weight $(23 \mathrm{~g})$ at the dose of $\mathrm{mPPZ} 0.2 \mu \mathrm{mol} / \mathrm{kg}$ to evaluate the antitumor effect. The tumor volume of mice given normal saline as control was grown continuously (Figure 6A). Through daily illumination, the trend of tumor growth with $\mathrm{HmN}$ group declined significantly, which further verified the PDT effect of mPPZ. $\mathrm{HmDN}$ group had much smaller tumor volume than $\mathrm{HmN}$, 


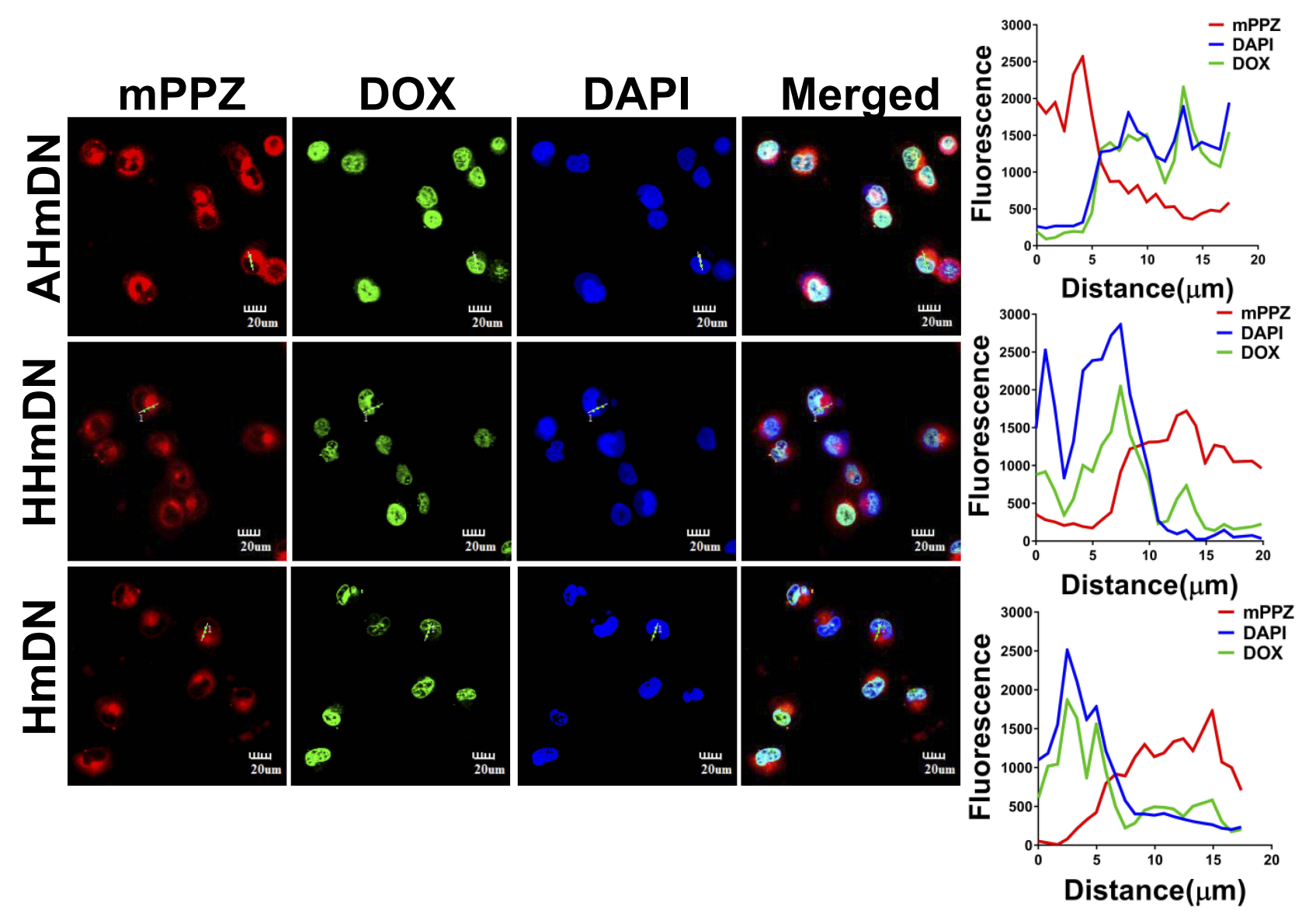

Figure 4 Cellular localization of $\mathrm{HmDN}, \mathrm{HHmDN}$ and $\mathrm{AHmDN}$ in $\mathrm{HI} 299$ cells and the fluorescence intensity profile.

Notes: After the incubation for $12 \mathrm{hrs}$ at the concentration of mPPZ $0.5 \mu \mathrm{M}$, HmDN, HHmDN and AHmDN could be absorbed into HI299 cells. DOX was mainly distributed in nucleus with nearly identical fluorescence intensity profile suggesting DOX was separated from nanoparticles. While mPPZ was mainly distributed in cytoplasm, not in nucleus.

which could be attributed to the combined effect of $\mathrm{mPPZ}$ and DOX. The slightly shrinked tumor volume in HHmDN group than HmDN group was probably caused by the enhanced targeting capability of HHmDN. Above all, AHmDN group had the lowest tumor growth rate (Figure 6A) and smallest tumor size (Figure 6C). The gap of tumor volume between $\mathrm{AHmDN}$ and $\mathrm{HHmDN}$ group was gradually enlarged after 2 days and there was 1.7 times difference at 7 days. The decreased tumor growth trend was as a result of the enhanced tumor targeting effect of AHmDN. After the treatment and measurement for 7 days, all the mice were killed and dissected to isolate the tumor tissue which was weighed to further verify the antitumor effect. The minimum tumor weight of AHmDN group was consistent with the result of tumor growth inhibition, reflecting strong antitumor activity (Figure 6B). The body weights of mice with treatment group were similar to the control group, demonstrating no apparent side effect to the mice
(Figure S7). Furthermore, the survival rate of mice treated with AHmDN over 40 days was significantly enhanced than $\mathrm{HmN}, \mathrm{HmDN}$ and HHmDN groups (Figure S8).

\section{Discussion}

Over the last four decades, photodynamic therapy (PDT) has been proved to be effective in the treatment of cancers, including superficial bladder cancer, early and obstructive lung cancer, skin cancer, Barrett's esophagus, head and neck cancers. ${ }^{31}$ In PDT, photosensitizers are accumulated at the tumor site and are illuminated with a light of specific wavelength to generate cytotoxic reactive oxygen species (ROS) to kill tumor cells. Compared with chemotherapy and radiation therapy, PDT has little systemic toxic effects on the biological systems and has minimal invasiveness. ${ }^{32}$ Photosensitizers are the key factor for PDT and highefficiency photosensitizers can improve the effect of 


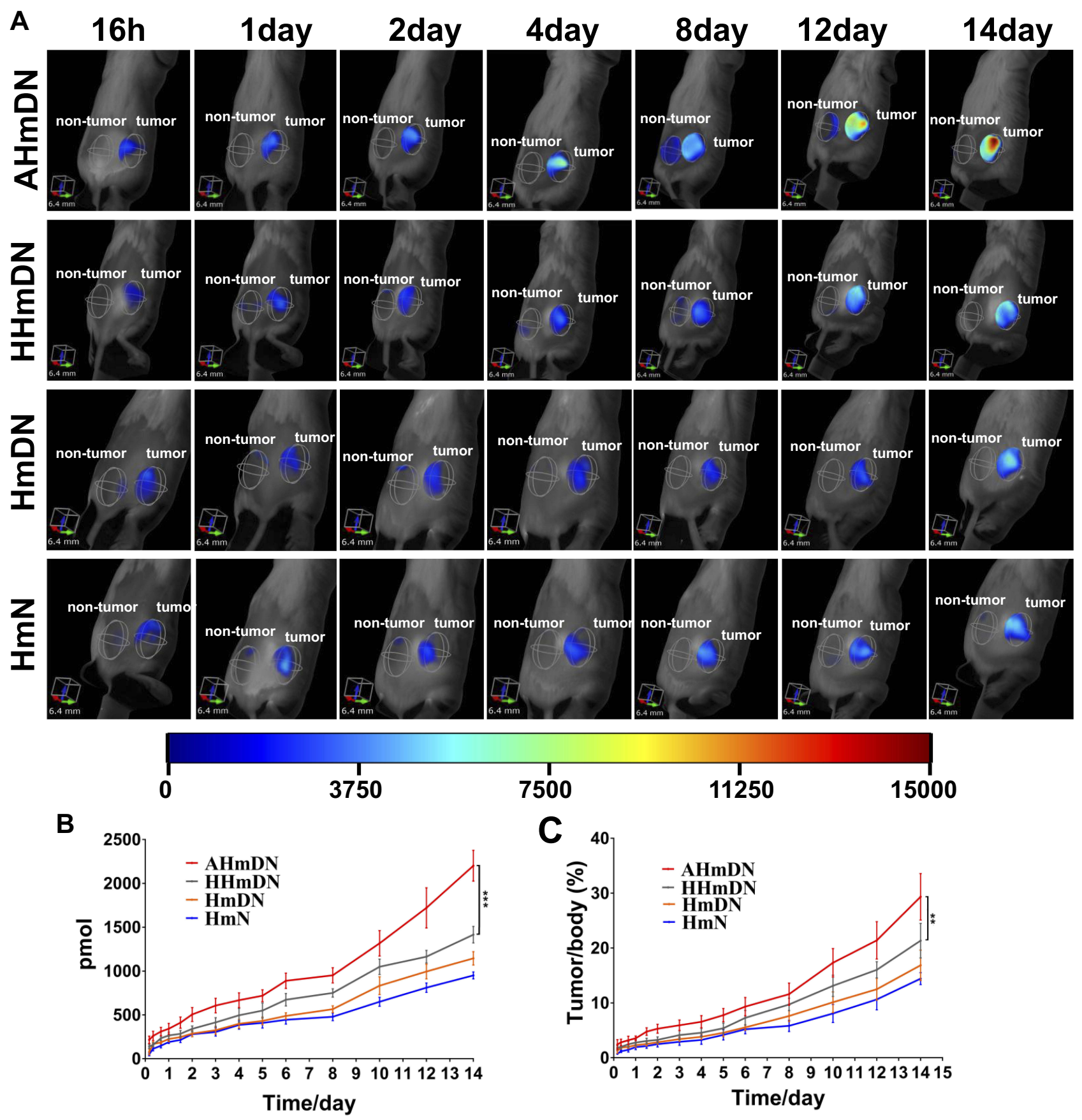

Figure 5 In vivo 3D fluorescent imaging and quantitative analysis of tumor-bearing mice injected with $\mathrm{HmN}, \mathrm{HmDN}, \mathrm{HHmDN}$ or AHmDN.

Notes: (A) Upon the injection of $\mathrm{mPPZ}$ at the dose of $0.2 \mu \mathrm{mol} / \mathrm{kg}$, all nanoparticles started to accumulate at the tumor site at I $2 \mathrm{hrs}$ and the fluorescence at tumor site (the left ROI was normal tissues, the right ROI was tumor site) in every group was increased constantly until 14 days. (B) The mPPZ content at tumor site was gradually elevated during the whole detection period in every group. The mPPZ content in AHmDN group was I.5 fold higher than HHmDN group at I4 days, which demonstrated an active targeting effect of $\mathrm{AHmDN}$. HmN group had less $\mathrm{mPPZ}$ at tumor site than other groups which results were consistent with cell uptake. (C) The mPPZ content ratio of tumor site and the whole body. The proportion of $\mathrm{mPPZ}$ at tumor site was continuously increased. AHmDN group had the highest ratio and approximately $30 \%$ of all mPPZ in the body accumulated in tumor site at 14 days. $* * * \mathrm{P}<0.00 \mathrm{I}, * * \mathrm{P}<0.01$.

PDT. Phthalocyanines, as second-generation photosensitizers, are characterized with strong absorption at far-red wavelengths and high quantum yields of singlet oxygen generation. ${ }^{33}$ Closed shell metal ions $\mathrm{Zn}^{2+}$ make phthalocyanines longer lifetimes of triplet state and higher triplet yields. ${ }^{34}$ Therefore, zinc phthalocyanine ( $\mathrm{ZnPc}$ ) has great application potential and is widely studied for PDT.

Hydrophobic $\mathrm{ZnPc}$ can permeate cell membranes easier than hydrophilic $\mathrm{ZnPc}$, and have enhanced therapeutic 
A

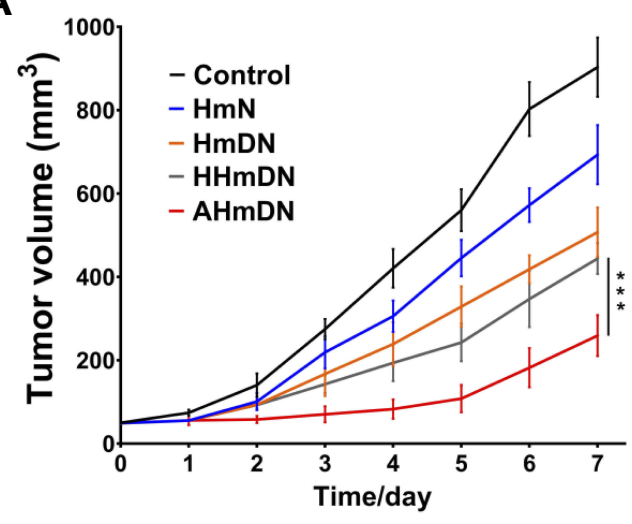

B

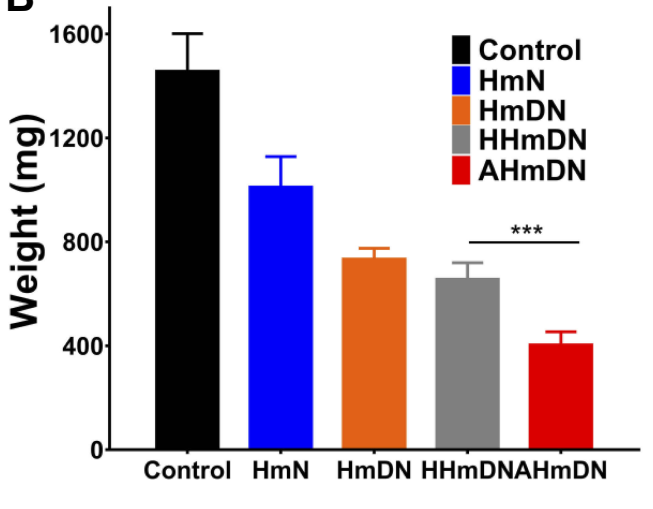

AHmDN HHmDN HmDN HmN Control

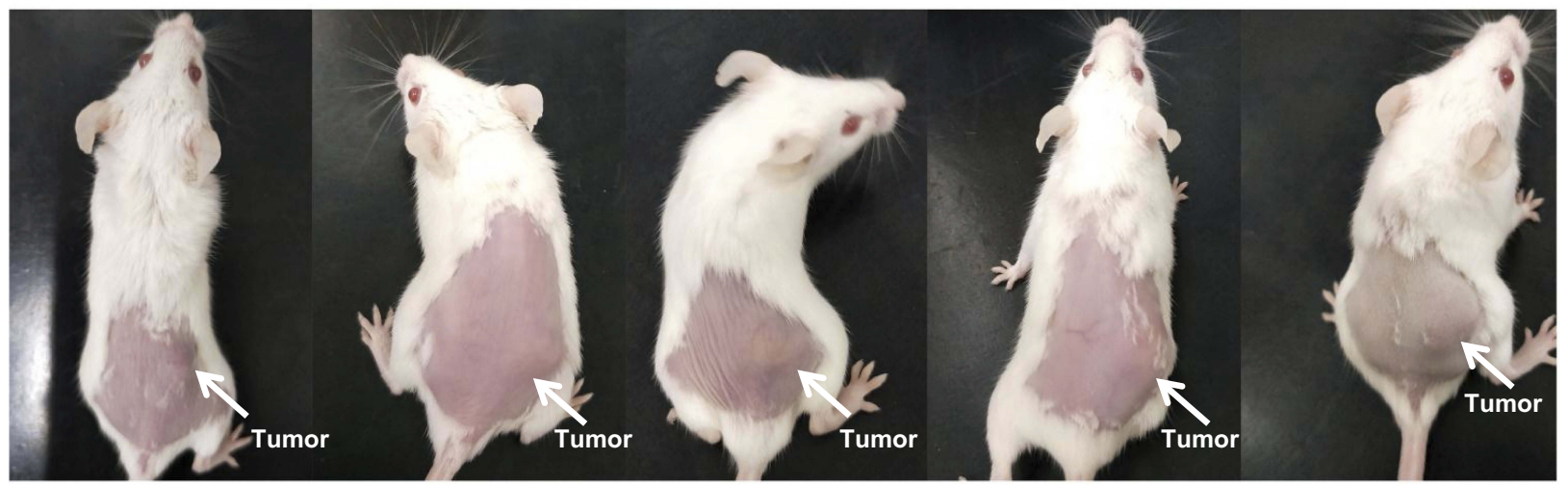

Figure 6 In vivo antitumor effect of $\mathrm{HmN}, \mathrm{HmDN}, \mathrm{HHmDN}$ and $\mathrm{AHmDN}$. H22 tumor-bearing mice were injected with $\mathrm{HmN}, \mathrm{HmDN}$, HHmDN or AHmDN in PBS solution at the dose of $\mathrm{mPPZ} 0.2 \mu \mathrm{mol} / \mathrm{kg}$.

Notes: (A) The tumor size was recorded accompanied by daily illumination $\left(50 \mathrm{~J} / \mathrm{cm}^{2}\right)$. Compared with control, all of them had antitumor effect to some extent and AHmDN group had the lowest tumor growth rate. (B) After the treatment for 7days, all the mice were executed and the weight of dissected tumors was recorded. AHmDN group had the lightest tumor than other groups which further indicated the enhanced antitumor effect. (C) Photographs of mice on the 7th day after the treatment with AhmDN, HHmDN, HmDN or $\mathrm{HmN}$. *** $\mathrm{P}<0.001$.

efficiency. However, the degree of aggregation between $\mathrm{ZnPc}$ in aqueous media is increased with their hydrophobicity. ${ }^{35}$ The ZnPc molecules' tendency to aggregate greatly influences their spectroscopic, photophysical and electrochemical properties in terms of fluorescence quenching ${ }^{36}$ and lower singlet oxygen generation ${ }^{37}$ which most probably due to enhanced state dissipation without radiation and reduced lifetime of the excited state. Some surfactants, such as cremophor EL or tween 20 , can make $\mathrm{ZnPc}$ disaggregated. But the use of surfactant is limited by its immunological side effects such as acute hypersensitivity and systemic immune reactions. ${ }^{38}$ Some solvents, such as dimethyl formamide, dimethyl sulfoxide, pyridine, coordinate to the central $\mathrm{Zn}^{2+}$ which inhibits the aggregation between $\mathrm{ZnPc}$ molecules. ${ }^{39}$ However, the aggregation is formed again once these solvents are diluted by aqueous solution. Therefore, limiting the aggregation of $\mathrm{ZnPc}$ is the key to develop high-efficiency photosensitizers.
HSA molecules had some hydrophobic pockets especially subdomains IIA and IIIA from X-ray structure analysis. ${ }^{40}$ These pockets could package hydrophobic photosensitizer mPPZ through hydrophobic interaction which could overcome the aggregation between $\mathrm{MPPZ}$ molecules. Compared with some surfactants leading phthalocyanine to non-aggregation by the interaction with micelle surface, ${ }^{41}$ HSA molecules, as a natural depolymerizing agent, had obvious advantages for its nontoxic and nonimmunogenic properties. Therefore, HSA was an ideal drug carrier for loading hydrophobic $\mathrm{ZnPc}$ molecules. However, after HSA was incubated with $\mathrm{mPPZ}$ $(100 \mu \mathrm{M})$ at different molar ratios (HSA:mPPZ was, respectively, $1: 2,1: 1,2: 1,4: 1,8: 1,20: 1)$ for $12 \mathrm{hrs}$, there was still no significant improvement of monomer absorption peak of mPPZ (Figure S1B). Dilution-incubation-purification (DIP) was an excellent method for loading hydrophobic $\mathrm{ZnPc}$ molecules. ${ }^{26}$ Hydrophobic ZnPc molecules, just like mPPZ, 
had a stronger tendency of aggregation with high concentration and the resulting aggregation had a larger particle size even leading to precipitate which hindered the interaction of HSA and mPPZ. Therefore, maintaining low concentration $(10 \mu \mathrm{M})$ was very essential during the preparation process. After the incubation adequately for $12 \mathrm{hrs}$, purification was another crucial step to remove unloaded mPPZ. DEAE anion exchange resin was used to purify HSA:mPPZ. Both HSA: $\mathrm{mPPZ}$ and unloaded $\mathrm{mPPZ}$ in Tris-HCl buffer solution (50mM NaCl, pH 8.5) could be adsorbed to DEAE column. HSA:mPPZ was eluted with high-concentration salt solution (Tris- $\mathrm{HCl}$ buffer solution, $300 \mathrm{mM} \mathrm{NaCl}, \mathrm{pH} 8.5$ ), while unloaded mPPZ was still attached to DEAE column. Thus, HSA:mPPZ was purified by this way.

Ethanol, as dehydrant, was used to agglomerate HSA molecules and their amino was crosslinked by glutaraldehyde to form stable nanoparticles. Except glutaraldehyde, mercaptoethanol was often used to crosslink HSA molecules by disrupting and recombining disulfide bonds of HSA. However, the transformation of disulfide bonds led to a great change of HSA structure. Because the loading capacity of HSA depended on its spatial structure, so mercaptoethanol was replaced by glutaraldehyde to crosslink HSA in order to prevent the release of mPPZ from HSA.

HSA was regarded as a chemical sponge which could bind many drugs. It had been reported that DOX could bind to HSA to form complex for improving the therapeutic effect of DOX. ${ }^{42}$ However, after HmN (containing HSA $30 \mathrm{mg}$ ) and DOX were incubated for $4 \mathrm{hrs}$ in $5 \mathrm{~mL}$ PBS at the concentration of DOX $30 \mu \mathrm{M}$, there was little DOX loading in $\mathrm{HmN}$. Hence, an improved method was used to load DOX into $\mathrm{HmN}$ by increasing the density of $\mathrm{HmN}$ and DOX $(500 \mu \mathrm{L}$ PBS, HSA 30mg, DOX 3mM). On the one hand, this increased the probability of interaction between DOX and HSA. On the other hand, DOX molecules in high concentration could generate aggregation ${ }^{42}$ which elevated hydrophobicity may increase the affinity for HSA. By this way, DOX was loaded into $\mathrm{HmN}$ and the stoichiometric ratio of HSA, mPPZ and DOX was about 1:0.8:5 (Figure S3).

Compared with healthy vessels in normal organs, tumors possessed leaky blood vessels and impaired lymphatic drainage, which allowed the selective access of nanoparticles to tumors, so-called enhanced permeability and retention (EPR) effect. ${ }^{43} \mathrm{HmDN}$ could target tumor tissue by EPR effect as a passive targeting strategy. For improving tumor targeting effect of HmDN, ATF peptide (amino-terminal fragment of urokinase), strong targeting ability to urokinase-type plasminogen activator receptor (UPAR) overexpressed in many types of cancer cells and tumor tissues, ${ }^{23}$ was used to modify HmDN. However, traditional way of conjugating biomacromolecules through covalent linkage, such as ester bond and amido bond, may disrupt the spatial structure of ATF which greatly reduced the targeting efficiency. In this study, ATFHSA, HSA molecule fused with ATF, was coupled to the surface of $\mathrm{HmDN}$ by the hydrophobic interaction between the HSA segment in ATF-HSA and HSA nanoparticles mediated by strong hydrophobic drug PTX. ATF still maintained its original target activity and may not be adhered to HSA nanoparticles by reason of the lack of hydrophobic domain for ATF. ${ }^{44}$ Therefore, AHmDN was successfully prepared using the simple and very effective method for coupling ATF to nanoparticles.

Flow cytometry analysis indicated that HmDN group had higher fluorescence intensity than $\mathrm{HmN}$ group (Figure 3A), which suggests more uptake in $\mathrm{HmDN}$ group. When the apoptosis occurred, apoptotic cells had some morphological variation including pyknosis, nuclear fragmentation and formation of apoptotic bodies. Whereas, cells presented necrotic features including the loss of cell membrane integrity in the late stages of apoptosis. ${ }^{45}$ Quantifications of the cell population showed cells of $\mathrm{HmDN}$ group in late apoptosis or necrosis were significantly more than $\mathrm{HmN}$ group (Figure 3C). The increased cell membrane permeability by reason of the loss of membrane integrity in $\mathrm{HmDN}$ group probably led to the enhanced uptake for nanoparticles.

Compared with healthy tissues, the abnormally wide fenestrations in the blood vessels allowed for the extravasation of materials with sizes up to several hundreds of nanometers in tumors. Together with the absence of lymphatic drainage, this brought to a relatively effective and selective accumulation of nanomedicines in tumors. The tumor accumulation of nanomedicines was mainly based on the EPR effect. Besides, the enhanced uptake of albumin-based nanoparticles in solid could be mediated by transcytosis initiated by binding of $60-\mathrm{kDa}$ glycoprotein (gp60) receptor on the surface of endothelial cells in tumor vasculature as well as binding of SPARC (secreted protein, acidic and rich in cysteine), an extracellular matrix glycoprotein overexpressed in a variety of cancers. ${ }^{46}$ These may be the reasons for the tumor targeting of $\mathrm{HmN}, \mathrm{HmDN}$, HHmDN and AHmDN. Whereas uPAR receptor-mediated tumor active targeting was reported to contribute more than HSA-mediated EPR effect, which was probably the reason why $\mathrm{AHmDN}$ had the enhanced tumor targeting than others. ${ }^{22}$ 
All the prepared nanoparticles had a certain degree of anticancer effect. However, the content of $\mathrm{mPPZ}$ in mice with photodynamic therapy was significantly reduced than that without illumination (Figure S9). Especially in $\mathrm{AHmDN}$ group, there was about threefold difference for mPPZ content at tumor site of the mice between treatment group and non-treatment group (raised in dark room). Besides the reduced tumor size, this could also be due to the photodegradation of phthalocyanine photosensitizers under strong light exposure. ${ }^{47}$ This suggested a method for further improving the therapeutic effect by increasing the injection frequency of the nanoparticles.

Since the occurrence and evolution of tumors was a complicated process, the combined administration with synergistic effects of multiple functional species usually exerted improved the antitumor effect in clinical cancer therapy ${ }^{48-51}$ DOX was a first-line chemotherapeutic agent for the treatment of a broad range of cancers through binding to topoisomerase enzyme II or directly intercalating with DNA to suppress the proliferation of tumor cells. ${ }^{52}$ Many studies reported that the combination of DOX and photosensitizers caused a synergistic cytotoxicity. ${ }^{53,54}$ Hence, many nanocarriers were used to deliver DOX and photosensitizers to tumor sites for improving antitumor effect, such as nanoparticles assembled by inorganic materials, ${ }^{55}$ polymeric micelles ${ }^{56}$ and liposomes. ${ }^{57}$ The covalent coupling of DOX or photosensitizers to the nanocarriers might affect their original mechanism of action. While simple mixing DOX and photosensitizers in nanocarriers might influence each other's activities by intermolecular interactions such as electrostatic force, Van der Waals interaction, hydrogen bonds and especially П-П stacking. In this study, disaggregated $\mathrm{ZnPc}$ was first loaded in hydrophobic pockets of HSA and then DOX was encapsulated in HSA nanoparticles. This ordered and separated loading in nanocarriers was positively beneficial for the therapeutic effect.

\section{Conclusions}

In summary, hydrophobic phthalocyanine zinc (mPPZ) was disaggregated by the interaction with HSA to generate complex HSA:mPPZ which fluorescence intensity was increased 10 fold than mPPZ in PBS. HSA:mPPZ was further crosslinked to nanoparticles $(\mathrm{HmN})$ simultaneously loading DOX (HmDN). HSA or ATF-HSA was assembled to the surface of HmDN using PTX as coupling agent to form two nanoparticles $\mathrm{HHmDN}$ or $\mathrm{AHmDN}$. All the four nanoparticles had well stability and $\mathrm{AHmDN}$ had higher cytotoxicity than others. Flow cytometry analysis showed tumor cell targeting property of $\mathrm{AHmDN}$ and more cells in late apoptotic or necrosis induced by $\mathrm{AHmDN}$. In vitro cell imaging detection indicated that DOX could enter into cell nucleus after the uptake of these nanoparticles while mPPZ in the cytoplasm. These nanoparticles had higher ROS production efficiency than $\mathrm{mPPZ}$ in the cells. In tumor-bearing mice, all the nanoparticles could be accumulated at tumor site continuously over 14 days. Most of all, AHmDN was demonstrated to have enhanced tumor targeting ability and antitumor effect than others. This drug delivery system could be used for carrying other hydrophobic $\mathrm{ZnPc}$ and chemotherapy drugs to improve the therapeutic effect of tumors.

\section{Acknowledgments}

Our research work is financially supported by grants from National Key R\&D Program of China (2017YFE0103200), Natural Science Foundation of China (31370737, 31400637, $31570745,31670739)$ and Natural Science Foundation of Shandong Province (ZR201808120006).

\section{Disclosure}

The authors report no conflicts of interest in this work.

\section{References}

1. Bray F, Ferlay J, Soerjomataram I, Siegel RL, Torre LA, Jemal A Global cancer statistics 2018: GLOBOCAN estimates of incidence and mortality worldwide for 36 cancers in 185 countries. CA Cancer J Clin. 2018;68(6):394-424. doi:10.3322/caac.21492

2. Pérez-Herrero E, Fernández-Medarde A. Advanced targeted therapies in cancer: drug nanocarriers, the future of chemotherapy. Eur J Pharm Biopharm. 2015;93:52-79. doi:10.1016/j.ejpb.2015.03.018

3. Yang K, Feng L, Liu Z. Stimuli responsive drug delivery systems based on nano-graphene for cancer therapy. Adv Drug Deliv Rev. 2016;105:228-241. doi:10.1016/j.addr.2016.05.015

4. Di Corato R, Béalle G, Kolosnjaj-Tabi J, et al. Combining magnetic hyperthermia and photodynamic therapy for tumor ablation with photoresponsive magnetic liposomes. ACS Nano. 2015;9(3):2904-2916. doi:10.1021/nn506949t

5. Chen H, Zhang X, Dai S, et al. Multifunctional gold nanostar conjugates for tumor imaging and combined photothermal and chemo-therapy. Theranostics. 2013;3(9):633-649. doi:10.7150/ thno. 6630

6. Liu T, Wang C, Cui W, et al. Combined photothermal and photodynamic therapy delivered by PEGylated MoS2 nanosheets. Nanoscale. 2014;6(19):11219-11225. doi:10.1039/C4NR03753G

7. Zha Z, Zhang S, Deng Z, Li Y, Li C, Dai Z. Enzyme-responsive copper sulphide nanoparticles for combined photoacoustic imaging, tumor-selective chemotherapy and photothermal therapy. Chem Commun. 2013;49(33):3455-3457. doi:10.1039/c3cc40608c

8. Devita VT Jr, Young RC, Canellos GP. Combination versus single agent chemotherapy: a review of the basis for selection of drug treatment of cancer. Cancer. 1975;35(1):98-110. doi:10.1002/(ISSN)10970142

9. Wang B-K, Yu X-F, Wang J-H, et al. Gold-nanorods-siRNA nanoplex for improved photothermal therapy by gene silencing. Biomaterials. 2016;78:27-39. doi:10.1016/j.biomaterials.2015.11.025 
10. Wan X, Min Y, Bludau H, et al. Drug combination synergy in worm-like polymeric micelles improves treatment outcome for small cell and non-small cell lung cancer. ACS Nano. 2018;12 (3):2426-2439. doi:10.1021/acsnano.7b07878

11. Shih Y-H, Peng C-L, Chiang P-F, Lin W-J, Luo T-Y, Shieh M-J. Therapeutic and scintigraphic applications of polymeric micelles: combination of chemotherapy and radiotherapy in hepatocellular carcinoma. Int J Nanomedicine. 2015;10:7443-7454. doi:10.2147/IJN

12. Zhang W, Shen J, Su H, et al. Co-delivery of cisplatin prodrug and chlorin e 6 by mesoporous silica nanoparticles for chemo-photodynamic combination therapy to combat drug resistance. ACS Appl Mater Interfaces. 2016;8(21):13332-13340. doi:10.1021/acsami.6b03881

13. Meng H, Mai WX, Zhang H, et al. Codelivery of an optimal drug/ siRNA combination using mesoporous silica nanoparticles to overcome drug resistance in breast cancer in vitro and in vivo. ACS Nano. 2013;7(2):994-1005. doi:10.1021/nn3044066

14. Zhao X, Chen Z, Zhao H, Zhang D, Tao L, Lan M. Multifunctional magnetic nanoparticles for simultaneous cancer near-infrared imaging and targeting photodynamic therapy. RSC Adv. 2014;4 (107):62153-62159. doi:10.1039/C4RA10801A

15. Gobbo OL, Sjaastad K, Radomski MW, Volkov Y, Prina-Mello A. Magnetic nanoparticles in cancer theranostics. Theranostics. 2015;5 (11):1249-1263. doi:10.7150/thno.11544

16. Bern M, Sand KMK, Nilsen J, Sandlie I, Andersen JT. The role of albumin receptors in regulation of albumin homeostasis: implications for drug delivery. $J$ Controlled Release. 2015;211:144-162. doi:10.1016/j.jconrel.2015.06.006

17. Tan YL, Ho HK. Navigating albumin-based nanoparticles through various drug delivery routes. Drug Discov Today. 2018;23 (5):1108-1114. doi:10.1016/j.drudis.2018.01.051

18. Ghuman J, Zunszain PA, Petitpas I, Bhattacharya AA, Otagiri M, Curry S. Structural basis of the drug-binding specificity of human serum albumin. $J$ Mol Biol. 2005;353(1):38-52. doi:10.1016/j.jmb. 2005.07.075

19. Hu Y-J, Ou-Yang Y, Dai C-M, Liu Y, Xiao X-H. Site-selective binding of human serum albumin by palmatine: spectroscopic approach. Biomacromolecules. 2009;11(1):106-112. doi:10.1021/bm 900961e

20. Elsadek B, Kratz F. Impact of albumin on drug delivery - new applications on the horizon. $J$ Controlled Release. 2012;157 (1):4-28. doi:10.1016/j.jconrel.2011.09.069

21. Kinoshita R, Ishima Y, Chuang VTG, et al. Improved anticancer effects of albumin-bound paclitaxel nanoparticle via augmentation of EPR effect and albumin-protein interactions using S-nitrosated human serum albumin dimer. Biomaterials. 2017;140:162-169. doi:10.1016/j.biomaterials.2017.06.021

22. Li R, Zheng K, Yuan C, Chen Z, Huang M. Be active or not: the relative contribution of active and passive tumor targeting of nanomaterials. Nanotheranostics. 2017;1(4):346. doi:10.7150/ntno.19380

23. Yang L, Sajja HK, Cao Z, et al. uPAR-targeted optical imaging contrasts as theranostic agents for tumor margin detection. Theranostics. 2014;4(1):106. doi:10.7150/thno.7409

24. Huang J, Zhong X, Wang L, Yang L, Mao H. Improving the magnetic resonance imaging contrast and detection methods with engineered magnetic nanoparticles. Theranostics. 2012;2(1):86-102. doi:10.71 50/thno. 4006

25. Roncucci G, Fantetti L, De Filippis MP, Dei D, Jori G Substituted metal-phthalocyanines, their preparation and the use thereof. In: Google Patents; 2006.

26. Li R, Zheng K, Hu P, et al. A novel tumor targeting drug carrier for optical imaging and therapy. Theranostics. 2014;4(6):642. doi:10. $7150 /$ thno. 8527

27. Marupudi NI, Han JE, Li KW, Renard VM, Tyler BM, Brem H. Paclitaxel: a review of adverse toxicities and novel delivery strategies. Expert Opin Drug Saf. 2007;6(5):609-621. doi:10.1517/ 14740338.6.5.609
28. Abi-Habib RJ, Singh R, Liu S, Bugge TH, Leppla SH, Frankel AE. A urokinase-activated recombinant anthrax toxin is selectively cytotoxic to many human tumor cell types. Mol Cancer Ther. 2006;5 (10):2556-2562. doi:10.1158/1535-7163.MCT-06-0315

29. Thorn CF, Oshiro C, Marsh S, et al. Doxorubicin pathways: pharmacodynamics and adverse effects. Pharmacogenet Genomics. 2011;21 (7):440. doi:10.1097/FPC.0b013e32833ffb56

30. Banfi S, Caruso E, Buccafurni L, Ravizza R, Gariboldi M, Monti E. Zinc phthalocyanines-mediated photodynamic therapy induces cell death in adenocarcinoma cells. J Organomet Chem. 2007;692 (6):1269-1276. doi:10.1016/j.jorganchem.2006.11.028

31. Lucky SS, Soo KC, Zhang Y. Nanoparticles in photodynamic therapy. Chem Rev. 2015;115(4):1990-2042. doi:10.1021/cr5004198

32. Dolmans DE, Fukumura D, Jain RK. Photodynamic therapy for cancer. Nature Rev Cancer. 2003;3(5):380. doi:10.1038/nrc1071

33. Mantareva V, Kussovski V, Angelov I, et al. Photodynamic activity of water-soluble phthalocyanine zinc (II) complexes against pathogenic microorganisms. Bioorg Med Chem. 2007;15(14):4829-4835. doi:10.1016/j.bmc.2007.04.069

34. Alberto ME, De Simone BC, Mazzone G, Sicilia E, Russo N. The heavy atom effect on $\mathrm{Zn}$ (II) phthalocyanine derivatives: a theoretical exploration of the photophysical properties. Phys Chem Chem Phys. 2015;17(36):23595-23601. doi:10.1039/C5CP03833B

35. Galstyan A, Riehemann K, Schäfers M, Faust A. A combined experimental and computational study of the substituent effect on the photodynamic efficacy of amphiphilic $\mathrm{Zn}$ (II) phthalocyanines. J Mater Chem B. 2016;4(34):5683-5691. doi:10.1039/C6TB01341D

36. Li X, Sinks LE, Rybtchinski B, Wasielewski MR. Ultrafast aggregate-toaggregate energy transfer within self-assembled light-harvesting columns of zinc phthalocyanine tetrakis (perylenediimide). $J$ Am Chem Soc. 2004;126(35):10810-10811. doi:10.1021/ja047176b

37. Kuznetsova NA, Gretsova NS, Derkacheva VM, Kaliya OL, Lukyanets EA. Sulfonated phthalocyanines: aggregation and singlet oxygen quantum yield in aqueous solutions. $J$ Porphyr Phthalocyanines. 2003;7(03):147-154. doi:10.1142/S1088424603000203

38. Weiszhár Z, Czúcz J, Révész C, Rosivall L, Szebeni J, Rozsnyay Z. Complement activation by polyethoxylated pharmaceutical surfactants: cremophor-EL, tween-80 and tween-20. Eur J Pharm Sci. 2012;45(4):492-498. doi:10.1016/j.ejps.2011.09.016

39. Ogunsipe A, Maree D, Nyokong T. Solvent effects on the photochemical and fluorescence properties of zinc phthalocyanine derivatives. J Mol Struct. 2003;650(1-3):131-140. doi:10.1016/S0022-2860(03) 00155-8

40. Bijelic A, Theiner S, Keppler BK, Rompel A. X-ray structure analysis of indazolium trans-[tetrachlorobis $(1 \mathrm{H}$-indazole) ruthenate (III)] (KP1019) bound to human serum albumin reveals two ruthenium binding sites and provides insights into the drug binding mechanism. J Med Chem. 2016;59(12):5894-5903. doi:10.1021/acs. jmedchem.6b00600

41. X-y L, He X, Ng AC, Wu C, Ng DK. Influence of surfactants on the aggregation behavior of water-soluble dendritic phthalocyanines. Macromolecules. 2000;33(6):2119-2123. doi:10.1021/ma9919110

42. Zheng K, Li R, Zhou X, et al. Dual actions of albumin packaging and tumor targeting enhance the antitumor efficacy and reduce the cardiotoxicity of doxorubicin in vivo. Int J Nanomed. 2015;10:5327.

43. Hickey JW, Santos JL, Williford J-M, Mao H-Q. Control of polymeric nanoparticle size to improve therapeutic delivery. $J$ Controlled Release. 2015;219:536-547. doi:10.1016/j.jconrel.2015.10.006

44. Huai Q, Mazar AP, Kuo A, et al. Structure of human urokinase plasminogen activator in complex with its receptor. Science. 2006;311(5761):656-659. doi:10.1126/science.1121143

45. Eom Y-W, Kim MA, Park SS, et al. Two distinct modes of cell death induced by doxorubicin: apoptosis and cell death through mitotic catastrophe accompanied by senescence-like phenotype. Oncogene. 2005;24(30):4765. doi:10.1038/sj.onc. 1208627 
46. Kratz F. Albumin as a drug carrier: design of prodrugs, drug conjugates and nanoparticles. $J$ Controlled Release. 2008;132 (3):171-183. doi:10.1016/j.jconrel.2008.05.010

47. Nyokong T, Antunes E. Photochemical and photophysical properties of metallophthalocyanines. In: Karl M Kadish, Kevin M Smith, Roger Guilard, editors. Handbook of Porphyrin Science (Volume 7) with Applications to Chemistry, Physics, Materials Science, Engineering, Biology and Medicine. World Scientific; 2010:247-357.

48. Lopez JS, Banerji U. Combine and conquer: challenges for targeted therapy combinations in early phase trials. Nature Rev Clin Oncol. 2017;14(1):57. doi:10.1038/nrclinonc.2016.96

49. Fan W, Yung B, Huang $P$, et al. Nanotechnology for multimodal synergistic cancer therapy. Chem Rev. 2017;117(22):13566-13638. doi:10.1021/acs.chemrev.7b00258

50. Chen Q, Wang X, Wang C, et al. Drug-induced self-assembly of modified albumins as nano-theranostics for tumor-targeted combination therapy. ACS Nano. 2015;9(5):5223-5233. doi:10.1021/acsnano. 5 b00640

51. Chen Q, Feng L, Liu J, et al. Intelligent albumin-mnO2 nanoparticles as $\mathrm{pH}-/ \mathrm{H} 2 \mathrm{O} 2$-responsive dissociable nanocarriers to modulate tumor hypoxia for effective combination therapy. Adv Mater. 2016;28 (33):7129-7136. doi:10.1002/adma.201601902
52. Zhang P, Li J, Ghazwani M, et al. Effective co-delivery of doxorubicin and dasatinib using a PEG-Fmoc nanocarrier for combination cancer chemotherapy. Biomaterials. 2015;67:104-114. doi:10.1016/j. biomaterials.2015.07.027

53. Hu DR, Chen LJ, Qu Y, et al. Oxygen-generating hybrid polymeric nanoparticles with encapsulated doxorubicin and chlorin e6 for trimodal imaging-guided combined chemo-photodynamic therapy. Theranostics. 2018;8(6):1558. doi:10.7150/thno.22989

54. Liao JF, Li WT, Peng JR, et al. Combined cancer photothermal-chemotherapy based on doxorubicin/gold nanorod-loaded polymersomes. Theranostics. 2015;5(4):345. doi:10.7150/thno.10731

55. Zeng L, Pan Y, Tian Y, et al. Doxorubicin-loaded NaYF4: yb/Tm-tiO2 inorganic photosensitizers for NIR-triggered photodynamic therapy and enhanced chemotherapy in drug-resistant breast cancers. Biomaterials. 2015;57:93-106. doi:10.1016/j.biomaterials.2015.04.006

56. Gao D, Lo PC. Polymeric micelles encapsulating pH-responsive doxorubicin prodrug and glutathione-activated zinc (II) phthalocyanine for combined chemotherapy and photodynamic therapy. $J$ Controlled Release. 2018;282:46-61. doi:10.1016/j.jconrel.2018.04.030

57. Hashemi M, Omidi M, Muralidharan B, et al. Layer-by-layer assembly of graphene oxide on thermosensitive liposomes for photo-chemotherapy. Acta Biomater. 2018;65:376-392. doi:10.1016/j.actbio.2017.10.040
International Journal of Nanomedicine

\section{Publish your work in this journal}

The International Journal of Nanomedicine is an international, peerreviewed journal focusing on the application of nanotechnology in diagnostics, therapeutics, and drug delivery systems throughout the biomedical field. This journal is indexed on PubMed Central, MedLine, CAS, SciSearch ${ }^{\circledR}$, Current Contents ${ }^{\mathbb{R}} /$ Clinical Medicine,

\section{Dovepress}

Journal Citation Reports/Science Edition, EMBase, Scopus and the Elsevier Bibliographic databases. The manuscript management system is completely online and includes a very quick and fair peer-review

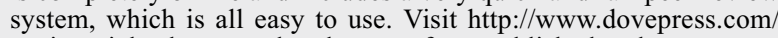
testimonials.php to read real quotes from published authors. 